\title{
Viable Control of an Epidemiological Model
}

\author{
Michel De Lara* Lilian Sofia Sepulveda Salcedo ${ }^{\dagger}$
}

March 3, 2022

\begin{abstract}
In mathematical epidemiology, epidemic control often aims at driving the number of infected individuals to zero, asymptotically. However, during the transitory phase, the number of infected can peak at high values. In this paper, we consider mosquito vector control in the Ross-Macdonald epidemiological model, with the goal of capping the proportion of infected by dengue at the peak. We formulate this problem as one of control of a dynamical system under state constraint. We allow for time-dependent fumigation rates to reduce the population of mosquito vector, in order to maintain the proportion of infected individuals by dengue below a threshold for all times. The so-called viability kernel is the set of initial states (mosquitoes and infected individuals) for which such a fumigation control trajectory exists. Depending on whether the cap on the proportion of infected is low, high or medium, we provide different expressions of the viability kernel. We also characterize so-called viable policies that produce, at each time, a fumigation rate as a function of current proportions of infected humans and mosquitoes, such that the proportion of infected humans remains below a threshold for all times. We provide a numerical application in the case of control of a dengue outbreak in 2013 in Cali, Colombia.
\end{abstract}

Keywords: epidemiology; control theory; viability theory; Ross-Macdonald model; dengue.

\footnotetext{
*Université Paris-Est, CERMICS (ENPC), 6-8 Avenue Blaise Pascal, Cité Descartes, 77455 Marne-la-Vallée, France, delara@cermics.enpc.fr

†Universidad Autónoma de Occidente, Km. 3 vía Cali-Jamundí, Cali, Colombia, lssepulveda@uao.edu.co
} 


\section{Contents}

1 Introduction 3

2 The viability problem 4

2.1 The Ross-MacDonald model . . . . . . . . . . . . . . . . . 4

2.2 Capping the proportion of infected humans . . . . . . . . 5

2.3 Viability kernel and viability domains . . . . . . . . 6 6

2.4 Viable equilibria . . . . . . . . . . . . . . . . . . 9

3 Characterization of the viability kernel $\mathbf{1 0}$

3.1 When the infected humans upper bound is low . . . . . . . . . 10

3.2 When the infected humans upper bound is high . . . . . . . 11

3.3 When the infected humans upper bound is medium . . . . . . 13

3.4 Epidemiological interpretation . . . . . . . . . . . . 28

3.5 Viable controls . . . . . . . . . . . . . . . . 31

4 Conclusion 32

A Appendix 34

A.1 Recalls on comparison theorems for differential systems . . . . 34

A.2 Epidemic model adjusted to 2013 dengue outbreak in Cali,

Colombia ................. . . . . . . . . . . 


\section{Introduction}

We consider vector control of the spread of an epidemic transmitted by vector in a Ross-Macdonald model. Our approach aims at controlling the number infected at the peak: we look for a trajectory of time-dependent vector mortality rates that is able to maintain the number of infected individuals below a threshold for all times. This approach differs from the widespread stationary control strategies, based upon having control reproductive number stricly less than one to ensure convergence, and also from cost minimization optimal control ones.

Indeed, many studies on mathematical modeling of infectious diseases consist of analyzing the stability of the equilibria of a differential system (behavioral models such as SIR, SIS, SEIR [9]). Those studies focus on asymptotic behavior and stability, generally leaving aside the transient behavior of the system, where the infection can reach high levels. In many epidemiological models, a significant quantity is the "basic reproductive number" $\mathcal{R}_{0}$ which depends on parameters such as the transmission rate, the death and birth rate, etc. Numerous works (see references in [19, 14]) exhibit conditions on $\mathcal{R}_{0}$ such that the number of infected individuals tends towards zero. With this tool, different (time-stationary) management strategies of the propagation of the infection - quarantine, vaccination, etc. - are compared with respect to how they modify $\mathcal{R}_{0}$. Thus, strategies are compared as to their capacity to drive the number of infected towards zero, focusing on asymptotics. However, during the transitory phase, the number of infected can peak at high values.

Other works deal with the whole trajectory, as in dynamic optimization where strategies are compared with respect to intertemporal costs and benefits [20], [23], [18], 22], [12], etc. More recently, [17] studies controls that minimize the outbreak size (or infectious burden) under the assumption that there are limited control resources.

Our approach focuses both on transitories and asymptotics, in a robust way. Instead of aiming at an equilibrium or optimizing, we look for policies able to maintain the infected individuals below a threshold for all times. To our knowledge, this approach is new in mathematical epidemiology. We have only found it mentioned in passing in [20] as a constraint - bounding above the maximum number infected at the peak - in a dynamic optimization problem, solved numerically.

In this paper, we formulate the problem as one of viability. In a nutshell, 
viability theory examines when and how can the state of a control system can be maintained within a given region for all times [2]. Such problems of dynamic control under constraints also refer to invariance issues [10]. In the control theory literature, problems of constrained control lead to the study of positively invariant sets, particularly ellipsoidal and polyhedral ones for linear systems (see [5], [15], [16] and the survey paper [6]); reachability of target sets or tubes for nonlinear discrete time dynamics is examined in [4. In continuous time, such a viability approach has been applied to models related to the sustainable management of fisheries [3], to viable strategies to ensure survival of some species [8], to secure the prey predator system [7], etc. In discrete-time, different examples can be found in [13] for sustainable management applications of viability.

The paper is organized as follows. In Section 2, we state the viability problem for the Ross-Macdonald model with control on the mosquito population by requiring to keep the proportion of infected humans below a given threshold. The viability kernel is the set of initial states such that there exists at least one fumigation control trajectory such that the resulting proportion of infected humans remains below a given threshold for all times. In Section 3, we provide a characterization of the viability kernel depending on whether the cap for the proportion of infected humans is low, high or medium. We also discuss viable controls. We apply our theoretical results to the case of the dengue outbreak in 2013 in Cali, Colombia. Thanks to numerical data provided by the Municipal Secretariat of Public Health of Cali, we provide figures of viability kernels and of viable trajectories.

\section{The viability problem}

First, we present the Ross-MacDonald model. Second, we formulate the viability problem, which consists in capping the proportion of infected humans using the dynamics described by the Ross-MacDonald model. Then, we introduce the viability kernel and viability domains. Finally, we describe viable equilibria, that are part of the viability kernel.

\subsection{The Ross-MacDonald model}

Different types of Ross-MacDonald models have been published [28]. We choose the one in [1], where both total populations (humans, mosquitoes) 
are normalized to 1 and divided between susceptibles and infected. The basic assumptions of the model are the following.

i) The human population $\left(N_{h}\right)$ and the mosquito population $\left(N_{m}\right)$ are closed and remain stationary.

ii) Humans and mosquitoes are homogeneous in terms of susceptibility, exposure and contact.

iii) The incubation period is ignored, in humans as in mosquitoes.

iv) Death induced by the disease is ignored, in humans as in mosquitoes.

v) Once infected, mosquitoes never recover.

vi) Only susceptibles get infected, in humans as in mosquitoes.

vii) Gradual immunity in humans is ignored.

Let $m(t)$ denote the proportion of infected mosquitoes at time $t$, and $h(t)$ the proportion of infected humans at time $t$. Therefore, $1-m(t)$ and $1-h(t)$ are the respective proportions of susceptibles. The Ross-MacDonald model is the following differential system

$$
\begin{aligned}
\frac{d m}{d t} & =\alpha p_{m} h(1-m)-\delta m, \\
\frac{d h}{d t} & =\alpha p_{h} \frac{N_{m}}{N_{h}} m(1-h)-\gamma h,
\end{aligned}
$$

where the parameters $\alpha, p_{m}, p_{h}, \xi=\frac{N_{m}}{N_{h}}, \delta$ and $\gamma$ are given in Table 1 .

\subsection{Capping the proportion of infected humans}

We turn the dynamical system (1) into a control system by replacing the natural death rate $\delta$ for mosquitoes in (1a) by a piecewise continuous function $u(\cdot): t \rightarrow u(t) \in[\underline{u}, \bar{u}]$, with $\underline{u}=\delta$. The function $u(\cdot)$ is the control on mosquito population that affects the mortality rate by fumigation of insecticides. The upper bound $\bar{u}$ is the maximal fumigation mortality rate. For notational simplicity, we put

$$
A_{m}=\alpha p_{m}, A_{h}=\alpha p_{h} \frac{M}{H}
$$




\begin{tabular}{|l|l|}
\hline Parameter & Description \\
\hline \hline$\alpha \geq 0$ & biting rate per time unit \\
\hline$\xi=N_{m} / N_{h} \geq 0$ & number of female mosquitoes per human \\
\hline $1 \geq p_{h} \geq 0$ & $\begin{array}{l}\text { probability of infection of a susceptible human } \\
\text { by infected mosquito biting }\end{array}$ \\
\hline $1 \geq p_{m} \geq 0$ & $\begin{array}{l}\text { probability of infection of a susceptible mosquito } \\
\text { when biting an infected human }\end{array}$ \\
\hline$\gamma \geq 0$ & recovery rate for humans \\
\hline$\delta \geq 0$ & (natural) death rate for mosquitoes \\
\hline
\end{tabular}

Table 1: Parameters of the Ross-MacDonald model (1)

Thinking about public health policies set by governmental entities, we impose the following constraint: the proportion $h(t)$ of infected humans must always remain below a threshold $\bar{H}$, where

$$
0<\bar{H}<1 \text {, }
$$

represents the maximum tolerated proportion of infected humans.

Therefore, the viability problem for the Ross-Macdonald model with control on the mosquito population is as follows. Given the control system

$$
\begin{aligned}
\frac{d m}{d t} & =A_{m} h(t)(1-m(t))-u(t) m(t), \\
\frac{d h}{d t} & =A_{h} m(t)(1-h(t))-\gamma h(t),
\end{aligned}
$$

determine if there exists a piecewise continuous control trajectory $u(\cdot)$ such that

$$
u(\cdot): t \mapsto u(t), \underline{u} \leq u(t) \leq \bar{u}, \forall t \geq 0,
$$

and such that the state trajectory given by (4) satisfies the viability constraint

$$
h(t) \leq \bar{H}, \quad \forall t \geq 0 .
$$

\subsection{Viability kernel and viability domains}

The solution to problem $(2.2)$ relies mainly on identifying the initial conditions, $(m(0), h(0))$, for the mosquitoes and infected humans, for which there 
exists a mortality rate due to fumigation, $u(\cdot)$ like (5), such that the solution (4) starting from $(m(0), h(0))$ satisfies (6). Such set of initial conditions is called the viability kernel.

Definition 1. The set of initial conditions $\left(m_{0}, h_{0}\right)$ for which there exists at least one fumigation policy (5) such that the solution to the system (4), with initial state $(m(0), h(0))=\left(m_{0}, h_{0}\right)$, satisfies the constraint (6) is called the viability kernel. We denote the viability kernel by $\mathbb{V}(\bar{H})$, that is,

$$
\mathbb{V}(\bar{H})=\left\{\begin{array}{l|l}
\left(m_{0}, h_{0}\right) & \begin{array}{c}
\text { there is } u(\cdot) \text { as in } \\
\text { such that the solution to } \\
\text { which starts from }\left(m_{0}, h_{0}\right) \\
\text { satisfies the constraint (6) }
\end{array}
\end{array}\right\} .
$$

The viability kernel is a subset of the constraint set

$$
\mathbb{V}^{0}(\bar{H})=\{(m, h) \mid 0 \leq m \leq 1,0 \leq h \leq \bar{H}\}=[0,1] \times[0, \bar{H}]
$$

that is,

$$
\mathbb{V}(\bar{H}) \subset \mathbb{V}^{0}(\bar{H})
$$

We define and present a geometric characterization of so-called viability domains of system (4), as they will be an important step to characterize the viability kernel.

Definition 2. A subset $\mathbb{V}$ of the set of states $[0,1] \times[0,1]$ is said to be a viability domain for the system (4) -(5) if there exists a control trajectory $u(\cdot)$ as in (5) such that the solution to (4), which starts from $(m(0), h(0)) \in \mathbb{V}$, remains within $\mathbb{V}$ for every $t \geq 0$.

Viability domains are related to the viability kernel as follows.

Theorem 3 ([2]). The viability kernel is the largest viability domain within the constraint set.

With system (4), we associate the vector field $\left(g_{m}, g_{h}\right)$ given by the two components

$$
\begin{aligned}
g_{m}(m, h, u) & =A_{m} h(1-m)-u m \\
g_{h}(m, h) & =A_{h} m(1-h)-\gamma h
\end{aligned}
$$


The system (4) is equivalent to

$$
\begin{aligned}
\frac{d m}{d t} & =g_{m}(m(t), h(t), u(t)), \\
\frac{d h}{d t} & =g_{h}(m(t), h(t)) .
\end{aligned}
$$

We now provide a geometric characterization of the viability domains of the system (11) with control constraints (5) using the vector field $\left(g_{m}, g_{h}\right)$. For this purpose, we first note that the system (11) is Marchaud [2] because:

- the constraint (5) on the controls is written as $u \in[\underline{u}, \bar{u}]$, where $[\underline{u}, \bar{u}]$ is closed;

- the components of the vector field $\left(g_{m}, g_{h}\right)$ in $(10)$ are continuous;

- the vector field $\left(g_{m}, g_{h}\right)$ and the set $[\underline{u}, \bar{u}]$ have linear growth (because the partial derivatives of $\left(g_{m}, g_{h}\right)$ are smooth and defined over the compact $[0,1] \times[0,1])$;

- the set $\left\{\left(g_{m}, g_{h}\right)(m, h, u) \mid u \in[\underline{u}, \bar{u}]\right\}$ is convex, for all $(m, h)$, because $g_{m}(m, h, u)$ is linearly dependent on the control $u$.

Second, we will use the following result to be found in [2] (Theorem 6.1.4, p. 203 and the remark p. 200).

Proposition 4. For a Marchaud controlled system, a closed subset $\mathbb{V}$ is viable if the tangent cone at any point in $\mathbb{V}$ contains at least one of the vectors in the family generated by the vector field at this point when the control varies.

In our case, we obtain the following geometric characterization of viability domains.

Proposition 5. Consider a closed subset $\mathbb{V}$ of $[0,1] \times[0,1]$. The set $\mathbb{V}$ is a viability domain for the system (4), if, whenever $(m, h)$ varies along the frontier $\partial \mathbb{V}$ of the set $\mathbb{V}$, there is a control $u \in[\underline{u}, \bar{u}]$ such that $\left(g_{m}(m, h, u), g_{h}(m, h)\right)$ is an inward-pointing vector, with respect to the set $\mathbb{V}$.

If the closed subset $\mathbb{V}$ has a piecewise smooth frontier $\partial \mathbb{V}$, it suffices — for the set $\mathbb{V}$ to be a viability domain for the system (4) - that the scalar product between the vector $\left(g_{m}, g_{h}\right)$ and a normal (non zero) outward-pointing vector (with respect to the set $\mathbb{V}$ ) be lower than or equal to zero. 


\section{$2.4 \quad$ Viable equilibria}

Control systems display a family of equilibria, indexed by stationary decisions. Within them, the equilibria which satisfy the constraints are part of the viability kernel: they are said to be viable equilibria.

\section{Stationary control: mortality rate due to constant fumigation}

Consider stationary mosquito control, that is, constant mortality rate due to fumigation:

$$
u(t)=u_{m}, \forall t \geq 0, \quad \text { with } \underline{u} \leq u_{m} \leq \bar{u} .
$$

The system (4) has the disease free equilibrium $(0,0)$ and, possibly, the endemic equilibrium point:

$$
E_{u_{m}}^{*}=\left(m^{*}, h^{*}\right)=\left(\frac{A_{m}-\gamma u_{m} / A_{h}}{A_{m}+u_{m}}, \frac{A_{h}-\gamma u_{m} / A_{m}}{A_{h}+\gamma}\right) .
$$

Such point $E_{u_{m}}^{*}$ exists in $[0,1]^{2}$ and has global asymptotic stability when

$$
0<A_{m} A_{h}-\gamma u_{m}
$$

The proof relies on the Poincaré-Bendixson theorem, excluding periodic orbits thanks to the Bendixson-Dulac criterion [30].

The viable equilibrium points $\left(m^{*}, h^{*}\right)$ are those for which $h^{*} \leq \bar{H}$. With (14), we deduce that the viable equilibrium points are the points (13) for which:

$$
0<\frac{A_{h}-\gamma u_{m} / A_{m}}{A_{h}+\gamma} \leq \bar{H}
$$

\section{Monotonicity properties}

The system (4) has monotonicity properties which will be practical for characterizing of the viability kernel.

Proposition 6. Let $(\bar{m}(t), \bar{h}(t))$ be the solution to (4) when $u(t)=\bar{u}$. If

$$
\bar{m}(0) \leq m(0), \bar{h}(0) \leq h(0)
$$

we have that

$$
\bar{m}(t) \leq m(t), \bar{h}(t) \leq h(t), \forall t>0
$$


Proof. Note that the components of vector field $\left(g_{m}, g_{h}\right)$ in 10 are smooth, and that, when $(m, h) \in[0,1]^{2}$,

$$
\frac{\partial g_{m}}{\partial h}=A_{m}(1-m) \geq 0, \frac{\partial g_{h}}{\partial m}=A_{h}(1-h) \geq 0 .
$$

By Definition 15 of Appendix A.1, $\left(g_{m}, g_{h}\right)$ is quasi monotonous in $(m, h)$ for any control $t \mapsto u(t)$.

Denote by $\left(\overline{g_{m}}, \overline{g_{h}}\right)$ the vector fields when $u=\bar{u}$ and $\underline{u}$, respectively, in $(10)$. Since $\overline{g_{m}} \leq g_{m}$ and $\overline{g_{h}} \leq g_{h}$, through the comparison Theorem 17 , we obtain the following the result: if $\bar{m}(0) \leq m(0)$ and $\bar{h}(0) \leq h(0)$, then $\bar{m}(t) \leq m(t)$ and $\bar{h}(t) \leq h(t)$, for every $t \geq 0$.

\section{Characterization of the viability kernel}

We will show that the characterization of the viability kernel (7) depends on whether the upper limit $\bar{H}$ for the proportion of infected humans in (6) is low, high or medium.

L) When $\bar{H}$ is low in (6), a strong constraint is put on the proportion of infected humans, and we will prove in $\$ 3.1$ that the viability kernel (7) reduces to the origin $\{(0,0)\}$.

H) When $\bar{H}$ is high in (6), hence allowing the proportion of infected humans to be large, the viability kernel is the entire constraint set $\mathbb{V}^{0}(\bar{H})$ in $(8)$, as we will show in 3.2 .

M) Finally, when $\bar{H}$ is medium in (6), which is a more interesting case, the viability kernel (7) is a strict subset of the constraint set $\mathbb{V}^{0}(\bar{H})$ in $(8)$, whose upper right frontier is a smooth curve that we characterize. The proof of this result will be given in $\S 3.3$.

\subsection{When the infected humans upper bound is low}

When the imposed constraint (6) is strong, meaning that the upper bound $\bar{H}$ for the proportion of infected humans is low, the viability kernel (7) reduces to the origin $\{(0,0)\}$ as follows. 
Proposition 7. If

$$
\bar{H}<\frac{A_{h}-\gamma \bar{u} / A_{m}}{A_{h}+\gamma},
$$

the viability kernel (7) only consists of the origin:

$$
\mathbb{V}(\bar{H})=\{(0,0)\} .
$$

Proof. First, note that the state $(0,0)$ is a viable equilibrium, as seen in $\$ 2.4$. Second, if the initial conditions $(m(0), h(0))$ are taken outside $\{(0,0)\}$, let us show that, for any $u(\cdot)$ as in (5), the solution to (4) violates the constraint (6).

Indeed, let $\bar{m}(t)$ and $\bar{h}(t)$ be solutions to (4) when $u(t)=\bar{u}$ and with initial state $(m(0), h(0))$. The assumption (19) implies the condition (14) that makes the endemic equilibrium point

$$
E_{\bar{u}}^{*}=\left(m_{\bar{u}}^{*}, h_{\bar{u}}^{*}\right)=\left(\frac{A_{m}-\gamma u_{m} / A_{h}}{A_{m}+u_{m}}, \frac{A_{h}-\gamma u_{m} / A_{m}}{A_{h}+\gamma}\right)
$$

exist and display global asymptotic stability, as seen in $\$ 2.4$. Hence, $\bar{h}(t)$ approaches $h_{\bar{u}}^{*}$ with $\bar{H}<h_{\bar{u}}^{*}$. So, by (19) and (21), we have $\bar{h}(t)>\bar{H}$ for all $t$ large enough.

From Proposition 6, we have that $\bar{h}(t) \leq h(t)$, for every $t \geq 0$, so, for a significantly large $t$, we have that $\bar{H}<\bar{h}(t) \leq h(t)$.

Therefore, for any initial condition outside $\{(0,0)\}$, for any $u(\cdot)$ as in (5), the solution to (4) violates the constraint (6) for times $t$ large enough, hence at least for one time.

\subsection{When the infected humans upper bound is high}

When the imposed constraint (6) is weak, meaning that the upper bound $\bar{H}$ for the proportion of infected humans is high, the viability kernel is the entire constraint set $\mathbb{V}^{0}(\bar{H})$ in $(8)$ as follows.

Proposition 8. If

$$
\frac{A_{h}}{A_{h}+\gamma} \leq \bar{H},
$$

the constraint set $\mathbb{V}^{0}(\bar{H})$ in (8) is strongly invariant and is, therefore, the viability kernel:

$$
\mathbb{V}(\bar{H})=\mathbb{V}^{0}(\bar{H})=[0,1] \times[0, \bar{H}] .
$$


Proof. Consider the vector field $\left(g_{m}, g_{h}\right)$ described in $(10)$, which corresponds to the system (4). We will study the vector $\left(g_{m}, g_{h}\right)$ along the four faces of the rectangle $\mathbb{V}^{0}(\bar{H})$ and we will prove that, for any control, $\left(g_{m}, g_{h}\right)$ is an inwardpointing vector, with respect to the set $\mathbb{V}^{0}(\bar{H})$ (that is, the vector $\left(g_{m}, g_{h}\right)$ belongs to the tangent cone, which is closed). Thanks to Proposition 4 (in fact, a time-varying extension), this suffices to prove that $\mathbb{V}^{0}(\bar{H})$ is strongly invariant.

- For any state $(m, h)$ on the vertical half-line $m=0$ and $h \geq 0$, we have that:

$$
g_{m}(0, h, u)=A_{m} h \geq 0, g_{h}(0, h)=-\gamma h \leq 0 .
$$

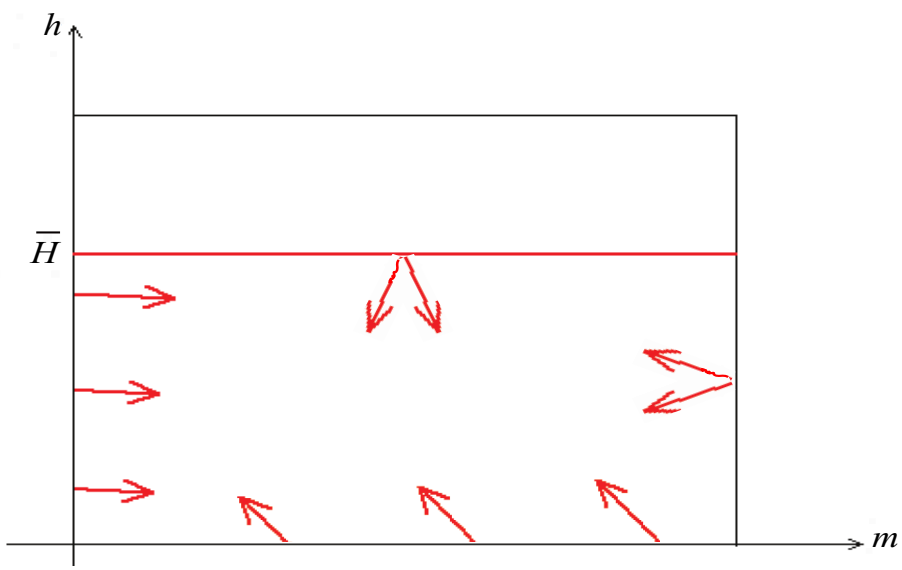

Figure 1: Vector field $\left(g_{m}, g_{h}\right)$ on the frontier of the constraint set $\mathbb{V}^{0}(\bar{H})$

Therefore, for any control $u \in[\underline{u}, \bar{u}]$, the vector $\left(g_{m}, g_{h}\right)$ always points towards the inside of $\mathbb{V}^{0}(\bar{H})$ (see the left part of Figure 1 ).

- For any state $(m, h)$ on the horizontal half-line $h=0$ and $m \geq 0$, we have that:

$$
g_{m}(0, h, u)=-u m \leq 0, g_{h}(0, h)=A_{h} m \geq 0 .
$$

Therefore, for any control $u \in[\underline{u}, \bar{u}]$, the vector $\left(g_{m}, g_{h}\right)$ always points towards the inside of $\mathbb{V}^{0}(\bar{H})$ (see the bottom part of Figure 1 ). 
- For any state $(m, h)$ on the vertical line $m=1$, we have that

$$
g_{m}(1, h, u)=-u \leq-\underline{u} \leq 0 .
$$

Therefore, for any control $u \in[\underline{u}, \bar{u}]$, the vector $\left(g_{m}, g_{h}\right)$ always points to the left of the line $m=1$. Hence, the vector $\left(g_{m}, g_{h}\right)$ always points towards the inside of $\mathbb{V}^{0}(\bar{H})$ (see the right part of Figure 1).

- Finally, for any state $(m, h)$ on the horizontal half-line $h=\bar{H}$ and $m \leq 1$, we have that

$$
g_{h}(m, \bar{H})=A_{h} m(1-\bar{H})-\gamma \bar{H} \leq A_{h}(1-\bar{H})-\gamma \bar{H} \leq 0,
$$

by (22) (which is equivalent to $\left.A_{h}(1-\bar{H}) \leq \gamma \bar{H}\right)$. Therefore, for any control $u \in[\underline{u}, \bar{u}]$, the vector $\left(g_{m}, g_{h}\right)$ always points below the line $h=\bar{H}$. Hence, the vector $\left(g_{m}, g_{h}\right)$ always points towards the inside of $\mathbb{V}^{0}(\bar{H})$ (see the top part of Figure 1 ).

Therefore, the constraint set $\mathbb{V}^{0}(\bar{H})$ is strongly invariant. So, the viability kernel is $\mathbb{V}^{0}(\bar{H})$ because every trajectory starting from $\mathbb{V}^{0}(\bar{H})$ remains in $\mathbb{V}^{0}(\bar{H})$, hence satisfies the constraint (6).

\subsection{When the infected humans upper bound is medium}

When the constraint is medium, that is, not too weak or too strong, then the upper bound for the proportion of infected humans is medium, and the viability kernel is in-between the origin $\{(0,0)\}$ and the entire constraint set $\mathbb{V}^{0}(\bar{H})$ in $(8)$. We introduce

$$
\bar{M}=\frac{\gamma \bar{H}}{A_{h}(1-\bar{H})},
$$

the value $m$ on the line $h=\bar{H}$ where the component $g_{h}(m, h)$ in $\left.10 \mathrm{~b}\right)$ is zero, that is,

$$
g_{h}(\bar{M}, \bar{H})=\bar{M}(1-\bar{H})-\gamma \bar{H}=0 .
$$

Proposition 9. If the upper bound for the proportion of infected humans is medium, that is, if

$$
\frac{A_{h}-\gamma \bar{u} / A_{m}}{A_{h}+\gamma}<\bar{H}<\frac{A_{h}}{A_{h}+\gamma},
$$


where

$$
0<\frac{A_{h}-\gamma \bar{u} / A_{m}}{A_{h}+\gamma}
$$

we have that $\bar{M}$, as defined in $(28)$ is such that $\bar{M}<1$, and the differential equation

$$
-g_{m}(m, \mathcal{H}(m), \bar{u}) \mathcal{H}^{\prime}(m)+g_{h}(m, \mathcal{H}(m))=0
$$

with initial condition

$$
\mathcal{H}(\bar{M})=\bar{H}
$$

has a unique nonnegative solution, and this solution is of the form

$$
\mathcal{H}:\left[\bar{M}, M_{\infty}\right] \rightarrow[0, \bar{H}] \text { with }\left\{\begin{array}{l}
\text { either } \quad \bar{M}<M_{\infty}<1 \text { and } \mathcal{H}\left(M_{\infty}\right)=0 \\
\text { or } \quad M_{\infty}=1
\end{array}\right.
$$

The viability kernel $(7)$ is $\mathbb{V}(\bar{H})=$

$$
([0, \bar{M}] \times[0, \bar{H}]) \bigcup\left\{(m, h) \mid \bar{M} \leq m \leq M_{\infty}, \quad 0 \leq h \leq \mathcal{H}(m)\right\}
$$

On the Figure 2, we display three viability kernels when the constraint is medium (corresponding to three values for the maximum tolerated proportion $\bar{H}$ of infected humans). 


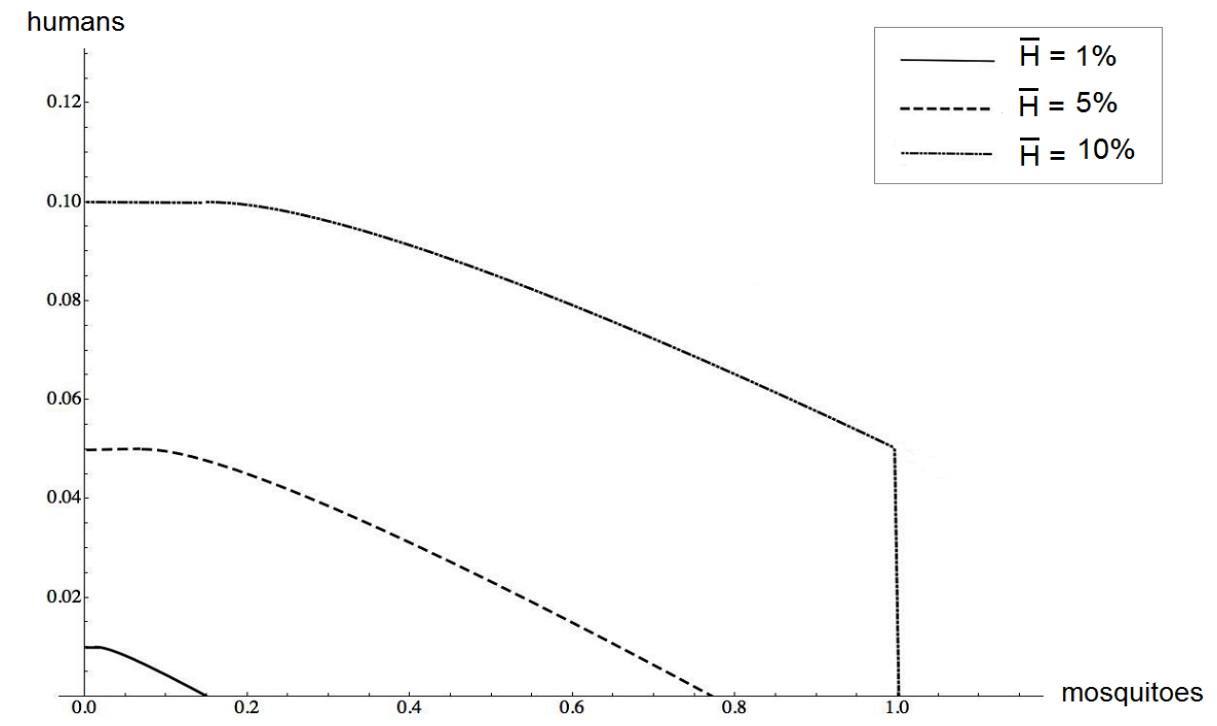

Figure 2: Three viability kernels for the Ross-MacDonald model (1), when the constraint is medium (corresponding to three values for the maximum tolerated proportion $\bar{H}$ of infected humans). Parameters are adjusted to the 2013 dengue outbreak in Cali, Colombia.

The proof consists of four lemmas. In Lemma 10, we describe the solution to 31a - 31b) (Lemma 11 provides additional information, that will be useful). Lemma 12 shows that the set

$$
\mathbb{V}=([0, \bar{M}] \times[0, \bar{H}]) \bigcup\left\{(m, h) \mid \bar{M} \leq m \leq M_{\infty}, \quad 0 \leq h \leq \mathcal{H}(m)\right\}
$$

is a viability domain. Finally, in Lemma 13 we prove that the set $\mathbb{V}$, defined in (34), is the largest viability domain within the constraint set (8), hence is the viability kernel by Theorem 3 .

Lemma 10. When the inequalities (30) are fulfilled, there exists a function $\mathcal{H}$, solution to the differential equation (31a) which satisfies the initial condition (31b) and has the form (32). The function $\mathcal{H}:\left[\bar{M}, M_{\infty}\right] \rightarrow$ $\left[\mathcal{H}\left(M_{\infty}\right), \bar{H}\right]$ is a strictly decreasing one-to-one mapping.

Proof. We conduct the proof in five steps. 
1. First, we note that

$$
\begin{aligned}
g_{m}(\bar{M}, \bar{H}, \bar{u}) & =A_{m} \bar{H}(1-\bar{M})-\bar{u} \bar{M} \text { by 10a } \\
& =A_{m} \bar{H}-\left(A_{m} \bar{H}+\bar{u}\right) \bar{M} \\
& =A_{m} \bar{H}-\left(A_{m} \bar{H}+\bar{u}\right) \frac{\gamma \bar{H}}{A_{h}(1-\bar{H})} \text { by definition (28) of } \bar{M} \\
& =\frac{\bar{H}}{A_{h}(1-\bar{H})}\left[A_{m} A_{h}(1-\bar{H})-\gamma\left(A_{m} \bar{H}+\bar{u}\right)\right] \\
& =\frac{\bar{H}}{A_{h}(1-\bar{H})}\left[A_{m} A_{h}-\gamma \bar{u}-\left(A_{m} A_{h}+\gamma A_{m}\right) \bar{H}\right] \\
& <0 \text { by (30b). }
\end{aligned}
$$

We easily deduce the following property, that will be useful later:

$$
m \geq \bar{M}, h \leq \bar{H} \Rightarrow g_{m}(M, H, \bar{u}) \leq g_{m}(\bar{M}, \bar{H}, \bar{u})<0 \text {. }
$$

2. Second, we show that there is a local solution to the differential equation 31a - 31b. Indeed, in the neighborhood of $(\bar{M}, \mathcal{H}(\bar{M}))=(\bar{M}, \bar{H})$, the coefficient $g_{m}(m, h, \bar{u})$ of $\mathcal{H}^{\prime}(m)$ in (31a) is negative, as we just saw it in the previous item 1 . Hence, in a neighborhood of $\bar{M}$, we can write $31 \mathrm{a}$ as

$$
\mathcal{H}^{\prime}(m)=\frac{g_{h}(m, \mathcal{H}(m))}{g_{m}(m, \mathcal{H}(m), \bar{u})}=\frac{A_{h} m(1-\mathcal{H}(m))-\gamma \mathcal{H}(m)}{A_{m} \mathcal{H}(m)(1-m)-\bar{u} m} .
$$

Applying the Cauchy-Lipschitz theorem to (36)-(31b), we know that there exists a unique local $C^{1}$ solution $\mathcal{H}$ defined on an interval $I$ around $\bar{M}$. We put

$$
I_{+}=I \cap[\bar{M},+\infty[.
$$

3. Third, we show that the local solution $\mathcal{H}: I_{+} \rightarrow \mathbb{R}$ is strictly decreasing in the neighborhood of $\bar{M}$. For this purpose, we will study the sign of $g_{h}(m, \mathcal{H}(m))$ for $m \approx \bar{M}$. We have that

$$
\begin{aligned}
g_{h}(m, \mathcal{H}(m)) & \left.=g_{h}(m, \mathcal{H}(m))-g_{h}(\bar{M}, \bar{H}) \text { by } 29\right) \\
& =A_{h} m(1-\mathcal{H}(m))-\gamma \mathcal{H}(m)-A_{h} \bar{M}(1-\bar{H})+\gamma \bar{H} \\
& =A_{h}(m-\bar{M})(1-\bar{H})+\left(A_{h} m+\gamma\right)(\bar{H}-\mathcal{H}(m))
\end{aligned}
$$


We have that $\mathcal{H}(m)-\bar{H}=o(m-\bar{M})$ when $m \rightarrow \bar{M}$. Indeed, the function $\mathcal{H}$ is $C^{1}$ and such that $\mathcal{H}^{\prime}(\bar{M})=0$, by $(36)$ since $g_{h}(\bar{M}, \bar{H})=0$ by (29). Therefore, we deduce that

$$
g_{h}(m, \mathcal{H}(m))=A_{h}(m-\bar{M})(1-\bar{H})+o(m-\bar{M})
$$

Hence, when $m>\bar{M}$ and $m \approx \bar{M}$, we have that $g_{h}(m, \mathcal{H}(m))>0$. Therefore, by (36) and item 1, the function $\mathcal{H}: I_{+} \rightarrow \mathbb{R}$ is strictly decreasing in a neighborhood of $\bar{M}$.

4. Fourth, we show that the local solution $\mathcal{H}: I_{+} \rightarrow \mathbb{R}$ is strictly decreasing. Indeed, let us suppose the contrary: there exists $m \in I_{+}, m>\bar{M}$, such that $\mathcal{H}^{\prime}(m)=0$. We denote by $\tilde{m}$ the smallest of such $m$. By item 3, we know that $\mathcal{H}^{\prime}(m)<0$ in the neighborhood of $\bar{M}$ (except at $\bar{M})$, so that $\tilde{m}>\bar{M}$. By definition of $\tilde{m}$, we have that $\mathcal{H}^{\prime}(m)<0$ for $m \in] \bar{M}, \tilde{m}[$. Hence, $\mathcal{H}(\tilde{m})<\mathcal{H}(\bar{M})=\bar{H}$, so that

$$
\begin{aligned}
g_{h}(\tilde{m}, \mathcal{H}(\tilde{m})) & =A_{h} \tilde{m}(1-\mathcal{H}(\tilde{m}))-\gamma \mathcal{H}(\tilde{m}) \text { by } 10 \\
& >A_{h} \bar{M}(1-\bar{H})-\gamma \bar{H} \\
& =g_{h}(\bar{M}, \bar{H})=0 \text { by } 29 .
\end{aligned}
$$

As a consequence, by the differential equation (36) and item 1, we obtain that $\mathcal{H}^{\prime}(\tilde{m})>0$. We arrive at a contradiction, since $\mathcal{H}^{\prime}(\tilde{m})=0$ by definition of $\tilde{m}$. Therefore, $\tilde{m}$ does not exist and the local solution $\mathcal{H}: I_{+} \rightarrow \mathbb{R}$ is strictly decreasing.

5. Finally, we show that the local solution $\mathcal{H}: I_{+} \rightarrow \mathbb{R}$ is such that $(32)$ holds true. For this purpose, we consider two cases.

- If, for every $m \in I_{+}, 0<\mathcal{H}(m)$, we show that $I_{+}=[\bar{M},+\infty[$. Indeed, as $\mathcal{H}$ decreases, we have $m \in I_{+} \Rightarrow 0<\mathcal{H}(m) \leq \mathcal{H}(\bar{M})=$ $\bar{H}$. Hence, we deduce that the solution $\mathcal{H}(m)$ to the equation (31a) exists over $m \in\left[\bar{M},+\infty\left[\right.\right.$. We denote $M_{\infty}=1$.

- If there exists an $m \in I_{+}$such that $\mathcal{H}(m)=0$, we denote by $\tilde{m}$ the smallest of such $m$. We have that $\mathcal{H}(\tilde{m})=0$ and, since $\mathcal{H}$ decreases, we have that $\mathcal{H}(\tilde{m})=0 \leq \mathcal{H}(m) \leq \mathcal{H}(\bar{M})=\bar{H}$ for every $m \in[\bar{M}, \tilde{m}]$.

We consider two subcases, as illustrated in Figures 3 and 4. 
- If $\tilde{m}<1$, we have that $\mathcal{H}(\tilde{m})=0$ and that $0 \leq \mathcal{H}(m) \leq \bar{H}$ for all $m \in[\bar{M}, \tilde{m}]$. We denote $M_{\infty}=\tilde{m}<1$.

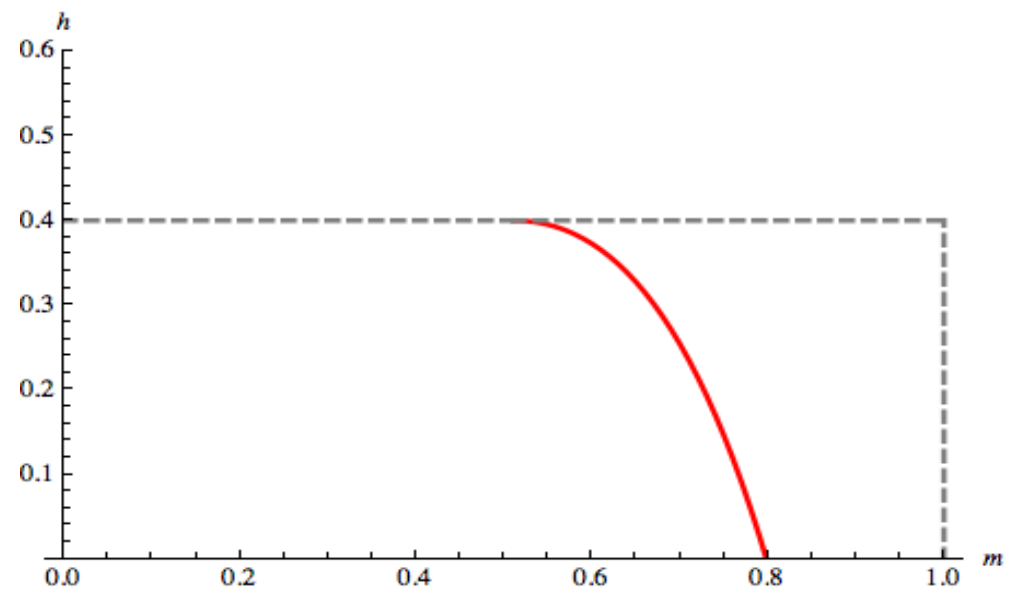

Figure 3: Case $M_{\infty}<1$. Solution graph for the differential equation (31a)(31b), with parameters $A_{h}=0.31066, A_{m}=0.02906, \gamma=0.1, \bar{u}=0.03733$ and $\bar{H}=0.4$

- if $\tilde{m} \geq 1$, we deduce that $0 \leq \mathcal{H}(m) \leq \bar{H}$ for every $m \in[\bar{M}, 1]$. We denote $M_{\infty}=1$.

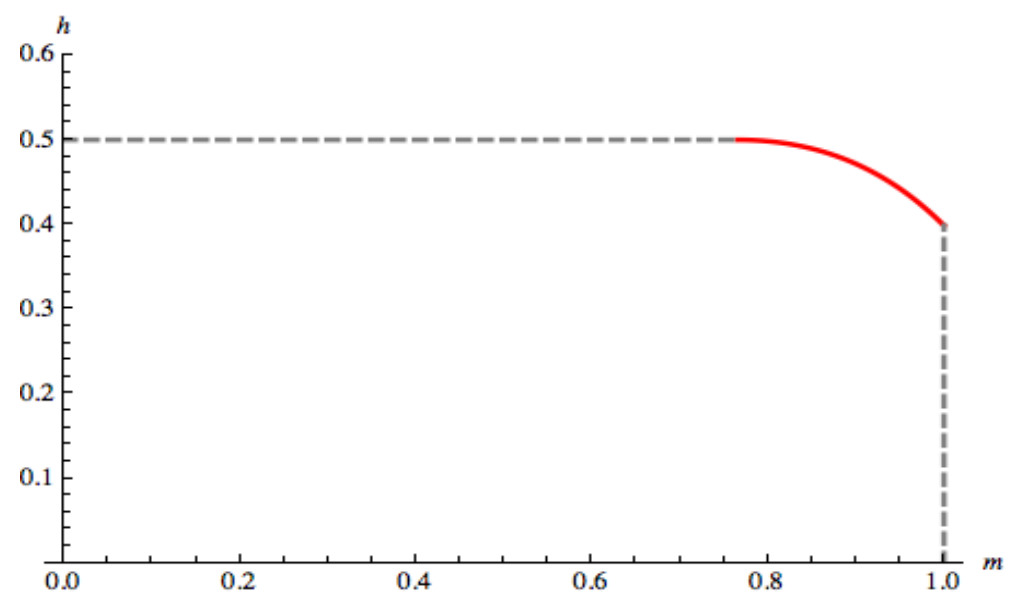

Figure 4: Case $M_{\infty}=1$. Solution graph for the differential equation (31a)(31b), with parameters $A_{h}=0.31066, A_{m}=0.02906, \gamma=0.1, \bar{u}=0.03733$ and $H=0.5$ 
Hence, we conclude that the solution to the differential equation (31a), which satisfies the initial condition (31b), is unique, has the form (32) and is strictly decreasing. As $\mathcal{H}(\bar{M})=\bar{H}$, the function $\mathcal{H}:\left[\bar{M}, M_{\infty}\right] \rightarrow\left[\mathcal{H}\left(M_{\infty}\right), \bar{H}\right]$ is a strictly decreasing one-to-one mapping.

We know show that the curve generated by the solution $\mathcal{H}$ is an orbit.

Lemma 11. The curve

$$
\left\{(m, \mathcal{H}(m)) \mid m \in\left[\bar{M}, M_{\infty}\right]\right\}
$$

is an orbit of the vector field the vector $\left(g_{m}, g_{h}\right)$, for the control $\bar{u}$. Indeed, for all

$$
\overline{\bar{m}}_{0} \in\left[\bar{M}, M_{\infty}\right] \text { and } \overline{\bar{h}}_{0}=\mathcal{H}\left(\overline{\bar{m}}_{0}\right) \in\left[\mathcal{H}\left(M_{\infty}\right), \bar{H}\right],
$$

there exists $T \geq 0$ such that the orbit of the trajectory $t \in[0, T] \mapsto(\overline{\bar{m}}(t), \overline{\bar{h}}(t))$ - the solution to (4) when $u(t)=\bar{u}$ and when the starting point is $(\overline{\bar{m}}(0), \overline{\bar{h}}(0))=$ $\left(\overline{\bar{m}}_{0}, \overline{\bar{h}}_{0}\right)-i$ included in the curve (39). In addition,

$$
\overline{\bar{m}}(T)=\bar{M} \text { and } \overline{\bar{h}}(T)=\bar{M} .
$$

Proof. When $\overline{\bar{m}}_{0}=\bar{M}$, then $\mathcal{H}\left(\overline{\bar{m}}_{0}\right)=\mathcal{H}(\bar{M})=\bar{H}$ by (31b), and the point $\left(\overline{\bar{m}}_{0}, \overline{\bar{h}}_{0}\right)=(\bar{M}, \bar{H})$ indeed belongs to the curve (31b). Therefore $T=0$.

Now, we suppose that $\overline{\bar{m}}_{0}>\bar{M}$, so that $\mathcal{H}\left(\overline{\bar{m}}_{0}\right)<\bar{H}$ because $\mathcal{H}$ is strictly decreasing by Lemma 10 . We define

$$
T=\inf \{t \geq 0 \mid \overline{\bar{m}}(t)<\bar{M} \text { or } \overline{\bar{h}}(t)>\bar{H}\},
$$

which is such that $T>0$, since $\overline{\bar{m}}(0)=\overline{\bar{m}}_{0}>\bar{M}$ and $\overline{\bar{h}}(0)=\mathcal{H}\left(\overline{\bar{m}}_{0}\right)<\bar{H}$.

We prove that $T<+\infty$. Since the inequality (30b) is fulfilled by assumption, the condition (14) is satisfied, so that the endemic equilibrium point $\left(m_{\bar{u}}^{*}, h_{u}^{*}\right)$ in (21) exists. By definition of an equilibrium point, we have that $g_{m}\left(m_{\bar{u}}^{*}, h_{\vec{u}}^{*}, \bar{u}\right)=0$. Therefore, as the left hand side of inequality (30a) can be restated as $h_{\bar{u}}^{*}<\bar{H}$, we deduce that $m_{u}^{*}<\bar{M}$, by (35). As seen in \$2.4, the endemic equilibrium point (21) displays global asymptotic stability. Therefore, $\overline{\bar{m}}(t) \rightarrow m_{\bar{u}}^{*}$ when $t \rightarrow+\infty$. As $m_{\bar{u}}^{*}<\bar{M}$, we deduce that there is a time $t$ such that $\overline{\bar{m}}(t)<\bar{M}$. As a consequence, $T$ as defined in 42 . is finite: $T<+\infty$. 
We now study the trajectory $t \in[0, T] \mapsto(\overline{\bar{m}}(t), \overline{\bar{h}}(t))$. By definition 42 of $T$, we have that

$$
\overline{\bar{m}}(T)=\bar{M} \text { or } \overline{\bar{h}}(T)=\bar{M}
$$

and that

$$
\overline{\bar{m}}(t) \geq \bar{M} \text { and } \overline{\bar{h}}(t) \leq \bar{H}, \quad \forall t \in[0, T] .
$$

By (35) and (43b), we deduce that

$$
\frac{d \overline{\bar{m}}(t)}{d t}=g_{m}(\overline{\bar{m}}(t), \overline{\bar{h}}(t), \bar{u})<0, \forall t \in[0, T] .
$$

As $\overline{\bar{m}}_{0} \leq M_{\infty}$ by $(40)$, we deduce that $\overline{\bar{m}}(t) \leq \overline{\bar{m}}_{0} \leq M_{\infty}$, for all $t \in[0, T]$. Together with $\overline{\bar{m}}(t) \geq \bar{M}$, we obtain that $\overline{\bar{m}}(t) \in\left[\bar{M}, M_{\infty}\right]$, for all $t \in$ $[0, T]$. Therefore, $\mathcal{H}(\overline{\bar{m}}(t))$ is well defined since $\mathcal{H}:\left[\bar{M}, M_{\infty}\right] \rightarrow\left[\mathcal{H}\left(M_{\infty}\right), \bar{H}\right]$ by 32 . We have, for all $t \in[0, T]$,

$$
\begin{aligned}
\frac{d}{d t}[\overline{\bar{h}}(t)-\mathcal{H}(\overline{\bar{m}}(t))]= & g_{m}(\overline{\bar{m}}(t), \overline{\bar{h}}(t), \bar{u})-\mathcal{H}^{\prime}(\overline{\bar{m}}(t)) g_{h}(\overline{\bar{m}}(t), \overline{\bar{h}}(t)) \\
& \text { by (4) and } 10 \\
& =0 \text { by } 31 \mathrm{a} .
\end{aligned}
$$

Therefore, for all $t \in[0, T]$,

$$
\overline{\bar{h}}(t)-\mathcal{H}(\overline{\bar{m}}(t))=\overline{\bar{h}}(0)-\mathcal{H}(\overline{\bar{m}}(0))=0 \text { by } 40,
$$

so that the trajectory $t \in[0, T] \mapsto(\overline{\bar{m}}(t), \overline{\bar{h}}(t))$ is included in the curve 39.

As a particular case, we have that $\overline{\bar{h}}(T)-\mathcal{H}(\overline{\bar{m}}(T))=0$. As $\overline{\bar{m}}(T)=\bar{M}$ or $\overline{\bar{h}}(T)=\bar{M}$ by 43a), we conclude that

$$
\overline{\bar{m}}(T)=\bar{M} \text { and } \overline{\bar{h}}(T)=\bar{M},
$$

since the function $\mathcal{H}:\left[\bar{M}, M_{\infty}\right] \rightarrow\left[\mathcal{H}\left(M_{\infty}\right), \bar{H}\right]$ is a strictly decreasing oneto-one mapping. We have proven (41).

Lemma 12. When the inequalities (30) are fulfilled, the set $\mathbb{V}$, defined in (34), is a viability domain for the system (4)-(5). 
Proof. Let the Hamiltonian $\mathcal{L}$ be defined, for every vector $\left(n_{m}, n_{h}\right)$, every state $(m, h)$ and control $u$, by

$$
\mathcal{L}\left(m, h, n_{m}, n_{h}, u\right)=g_{m}(m, h, u) n_{m}+g_{h}(m, h) n_{h} .
$$

The Hamiltonian is the scalar product between the vectors $\left(g_{m}, g_{h}\right)$ and $\left(n_{m}, n_{h}\right)$.

We will check that, for any given point along the piecewise-smooth frontier of the set $\mathbb{V}$, defined in (34), there is at least one control $u \in[\underline{u}, \bar{u}]$ such that the value (45) of the Hamiltonian is lower than or equal to zero when $\left(n_{m}, n_{h}\right)$ is a normal outward-pointing vector (with respect to the set $\mathbb{V}$ ). For kink points between two smooth parts, we will do the same but with the cone generated by two normal outward-pointing vectors, corresponding to each of the smooth parts.

We will divide the frontier of the set $\mathbb{V}$, defined in (34), in five or seven parts (see Figures 3 and 4 ) as follows.

(a) On the horizontal segment $\left\{(m, 0) \mid 0<m<M_{\infty}\right\}$, on the vertical segment $\{(0, h) \mid 0<h<\bar{H}\}$ and at the points $(0,0)$ and $(0, \bar{H})$, all vectors $\left(g_{m}, g_{h}\right)$ point towards the inside of the set $\mathbb{V}$, defined in (34). Indeed, it suffices to copy the proof of Proposition 8.

(b) Along the segment $\{(m, \bar{H}) \mid 0<m<\bar{M}\}$, a normal outward-pointing vector is

$$
\left(\begin{array}{c}
n_{m} \\
n_{h}
\end{array}\right)=\left(\begin{array}{l}
0 \\
1
\end{array}\right) .
$$

Therefore, for any control $u \in[\underline{u}, \bar{u}]$, the value (45) of the Hamiltonian is

$$
\mathcal{L}\left(m, \bar{H}, n_{m}, n_{h}, u\right)=g_{h}(m, \bar{H}) \times 1<0,
$$

by 29 and because $m<\bar{M}$.

(c) Along the curve $\left\{(m, \mathcal{H}(m)) \mid \bar{M}<m<M_{\infty}\right\}$, a normal outward-pointing vector is

$$
\left(\begin{array}{c}
n_{m} \\
n_{h}
\end{array}\right)=\left(\begin{array}{c}
-\mathcal{H}^{\prime}(m) \\
1
\end{array}\right) .
$$

Therefore, for the control $\bar{u}$, the value (45) of the Hamiltonian is

$$
\mathcal{L}\left(m, \bar{H}, n_{m}, n_{h}, \bar{u}\right)=-g_{m}(m, \mathcal{H}(m), \bar{u}) \mathcal{H}^{\prime}(m)+g_{h}(m, \mathcal{H}(m)) \times 1=0,
$$

because the function $\mathcal{H}$ is the solution to the differential equation 31a. 
(d) The point $(\bar{M}, \bar{H})$ is a kink, to which is attached a cone of normal vectors generated by the two following normal outward-pointing vectors.

- A normal outward-pointing vector to the horizontal segment $\{(m, \bar{H}) \mid 0 \leq$ $m \leq \bar{M}\}$ at the kink point $(\bar{M}, \bar{H})$ is

$$
\left(\begin{array}{c}
n_{m} \\
n_{h}
\end{array}\right)=\left(\begin{array}{l}
0 \\
1
\end{array}\right)
$$

Therefore, for any control $u \in[\underline{u}, \bar{u}]$, the value 45 of the Hamiltonian is

$$
\mathcal{L}\left(\bar{M}, \bar{H}, n_{m}, n_{h}, u\right)=g_{h}(\bar{M}, \bar{H}) \times 1=0 \text { by }(29) \text {. }
$$

- A normal outward-pointing vector to the curve $\{(m, \mathcal{H}(m)) \mid \bar{M} \leq$ $\left.m \leq M_{\infty}\right\}$ at the kink point $(\bar{M}, \bar{H})$ is

$$
\left.\left(\begin{array}{c}
n_{m} \\
n_{h}
\end{array}\right)=\left(\begin{array}{c}
-\mathcal{H}^{\prime}(M) \\
1
\end{array}\right)=\left(\begin{array}{l}
0 \\
1
\end{array}\right) \text { by } 36 \text { and } 29\right) \text {. }
$$

Therefore, for any control $u \in[\underline{u}, \bar{u}]$, the value $(45)$ of the Hamiltonian is

$$
\mathcal{L}\left(\bar{M}, \bar{H}, n_{m}, n_{h}, u\right)=g_{h}(\bar{M}, \bar{H}) \times 1=0 \text { by }(29) .
$$

We conclude that, for any control $u \in[\underline{u}, \bar{u}]$, the value 45 of the Hamiltonian is zero for any combination of the two normal outward-pointing vectors above. As a consequence, all vectors $\left(g_{m}, g_{h}\right)$ point towards the inside of the set $\mathbb{V}$, defined in (34), at the kink point $(\bar{M}, \bar{H})$.

(e) When $M_{\infty}<1$ in (32) (see Figure 3), there only remains to consider the kink point $\left(M_{\infty}, 0\right)$.

- A normal outward-pointing vector to the horizontal segment $\{(m, 0) \mid 0 \leq$ $\left.m \leq M_{\infty}\right\}$ at the kink point $\left(M_{\infty}, 0\right)$ is

$$
\left(\begin{array}{c}
n_{m} \\
n_{h}
\end{array}\right)=\left(\begin{array}{l}
1 \\
0
\end{array}\right)
$$

Therefore, for any control $u \in[\underline{u}, \bar{u}]$, the value 45 of the Hamiltonian is

$$
\mathcal{L}\left(M_{\infty}, 0, n_{m}, n_{h}, u\right)=g_{m}\left(M_{\infty}, 0, u\right) \times 1=-u M_{\infty} \leq-\underline{u} M_{\infty} \leq 0 .
$$


- A normal outward-pointing vector to the curve $\{(m, \mathcal{H}(m)) \mid \bar{M} \leq$ $\left.m \leq M_{\infty}\right\}$ at the kink point $\left(M_{\infty}, 0\right)$ is

$$
\left(\begin{array}{c}
n_{m} \\
n_{h}
\end{array}\right)=\left(\begin{array}{c}
-\mathcal{H}^{\prime}\left(M_{\infty}\right) \\
1
\end{array}\right) .
$$

Therefore, for the control $\bar{u}$, the value (45) of the Hamiltonian is

$$
\begin{aligned}
\mathcal{L}\left(M_{\infty}, 0, n_{m}, n_{h}, \bar{u}\right) & =-g_{m}\left(M_{\infty}, 0, \bar{u}\right) \mathcal{H}^{\prime}\left(M_{\infty}\right)+g_{h}\left(M_{\infty}, 0\right) \times 1 \\
& =0 \text { by 31a } .
\end{aligned}
$$

We conclude that, for the control $\bar{u}$, the value (45) of the Hamiltonian is zero for any combination of the two normal outward-pointing vectors above. As a consequence, the vector $\left(g_{m}, g_{h}\right)$, for the control $\bar{u}$, points towards the inside of the set $\mathbb{V}$, defined in (34), at the kink point $\left(M_{\infty}, 0\right)$.

(f) When $M_{\infty}=1$ in (32) (see Figure 4), we have to consider the vertical segment $\{(1, h) \mid 0 \leq h<\mathcal{H}(1)\}$. A normal outward-pointing vector to the vertical segment $\{(1, h) \mid 0 \leq h<\mathcal{H}(1)\}$ has the form

$$
\left(\begin{array}{c}
n_{m} \\
n_{h}
\end{array}\right)=\left(\begin{array}{l}
1 \\
0
\end{array}\right) \text {. }
$$

Therefore, for any control $u \in[\underline{u}, \bar{u}]$, the value (45) of the Hamiltonian is

$$
\mathcal{L}\left(1, h, n_{m}, n_{h}, u\right)=g_{m}(1, h, u) \times 1=-u \leq-\underline{u} \leq 0 .
$$

As a consequence, all vectors $\left(g_{m}, g_{h}\right)$ point towards the inside of the set $\mathbb{V}$, defined in (34), along the vertical segment $\{(1, h) \mid 0 \leq h<\mathcal{H}(1)\}$.

(g) When $M_{\infty}=1$ in (32) (see Figure 44, there remains to consider the kink point $(1, \mathcal{H}(1))$.

- A normal outward-pointing vector to the vertical segment $\{(1, h) \mid 0 \leq$ $h \leq \mathcal{H}(1)\}$ at the kink point $(1, \mathcal{H}(1))$ is

$$
\left(\begin{array}{c}
n_{m} \\
n_{h}
\end{array}\right)=\left(\begin{array}{l}
1 \\
0
\end{array}\right) \text {. }
$$

Therefore, for any control $u \in[\underline{u}, \bar{u}]$, the value 45 of the Hamiltonian is

$$
\mathcal{L}\left(1, \mathcal{H}(1), n_{m}, n_{h}, u\right)=g_{m}(1, \mathcal{H}(1), u) \times 1=-u \leq-\underline{u}<0 .
$$


- A normal outward-pointing vector to the curve $\{(m, \mathcal{H}(m)) \mid \bar{M}<$ $\left.m<M_{\infty}\right\}$ at the kink point $(1, \mathcal{H}(1))$ is

$$
\left(\begin{array}{c}
n_{m} \\
n_{h}
\end{array}\right)=\left(\begin{array}{c}
-\mathcal{H}^{\prime}(1) \\
1
\end{array}\right) .
$$

Therefore, for the control $\bar{u}$, the value 45 of the Hamiltonian is

$$
\begin{aligned}
\mathcal{L}\left(1, \mathcal{H}(1), n_{m}, n_{h}, \bar{u}\right) & =-g_{m}(1, \mathcal{H}(1), \bar{u}) \mathcal{H}^{\prime}(1)+g_{h}(1, \mathcal{H}(1)) \times 1 \\
& =0 \text { by } 31 \mathrm{a} .
\end{aligned}
$$

We conclude that, for the control $\bar{u}$, the value (45) of the Hamiltonian is zero for any combination of the two normal outward-pointing vectors above. As a consequence, the vector $\left(g_{m}, g_{h}\right)$, for the control $\bar{u}$, points towards the inside of the set $\mathbb{V}$, defined in (34), at the kink point $(1, \mathcal{H}(1))$.

From Proposition 5, we can conclude that $\mathbb{V}$, defined in (34), is a viability domain.

Lemma 13. When the inequalities (30) are fulfilled, the set $\mathbb{V}$, defined in (34), is the largest viability domain for the system (4), within the constraint set (8). 


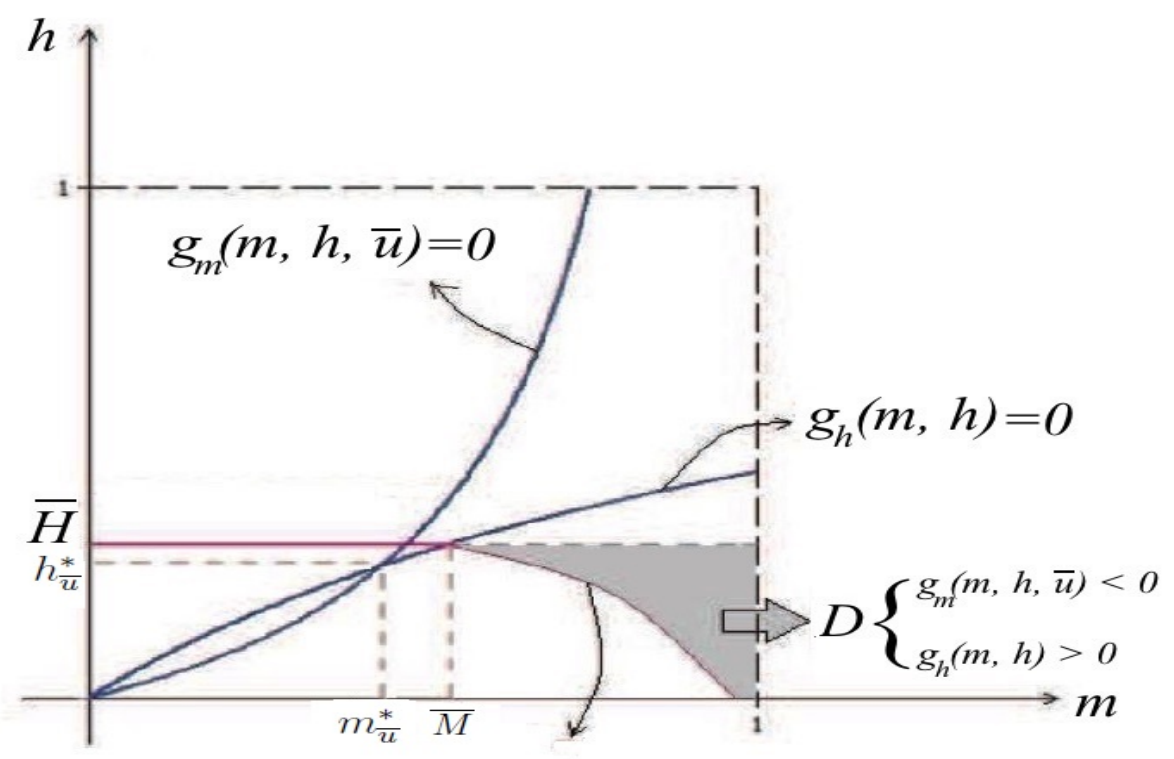

Figure 5: The shaded portion of the graph corresponds to the complement of the viability kernel $\mathbb{V}(\bar{H})$ with respect to the constraint set $\mathbb{V}^{0}(\bar{H})$, when $M_{\infty}<1$

Proof. We prove that, for any control trajectory $u(\cdot)$ as in (5), the trajectory $t \mapsto(m(t), h(t))$ solution of (4) and that starts from a point

$$
(m(0), h(0))=\left(m_{0}, h_{0}\right) \in \mathbb{V}^{0}(\bar{H}) \backslash \mathbb{V}
$$

does not satisfy the constraint (6) for at least one $t>0$. In Figure 5 , the set $\mathbb{V}^{0}(\bar{H}) \backslash \mathbb{V}$ is represented by the shaded part (in the case when $M_{\infty}<1$ ).

First, we examine the point $\left(m_{0}, h_{0}\right) \in \mathbb{V}^{0}(\bar{H}) \backslash \mathbb{V}$. On the one hand, as $\left(m_{0}, h_{0}\right) \in \mathbb{V}^{0}(\bar{H})$, we have that $0 \leq h_{0} \leq \bar{H}$. On the other hand, since $\left(m_{0}, h_{0}\right) \notin \mathbb{V}$, where the set $\mathbb{V}$ is defined in (34), we deduce from Lemma 10 , and especially from $(32)$, that

$$
\begin{aligned}
\left(m_{0}, h_{0}\right) \in \mathbb{V}^{0}(\bar{H}) \backslash \mathbb{V} & \Longleftrightarrow \bar{M}<m_{0} \leq 1,0 \leq h_{0} \leq \bar{H} \\
& \text { and } \begin{cases}\text { either } & m_{0}>M_{\infty}, \\
\text { or } & m_{0} \leq M_{\infty} \text { and } \mathcal{H}\left(m_{0}\right)<h_{0} .\end{cases}
\end{aligned}
$$


Notice that, if $h_{0}=\bar{H}$, then

$$
\begin{aligned}
\frac{d h(t)}{d t \quad \mid t=0} & =g_{h}\left(m_{0}, \bar{H}\right) \text { by (4) and }(10) \\
& >g_{h}(\bar{M}, \bar{H}) \text { by } 10 \text { and } \bar{M}<m_{0} \\
& =0 \text { by }(29) .
\end{aligned}
$$

Therefore, $h(t)>\bar{H}$, for $t>0$ small enough. As a consequence, we will concentrate on the case $h_{0}<\bar{H}$.

Second, we show that there exists a point $\left(\overline{\bar{m}}_{0}, \overline{\bar{h}}_{0}\right)$ such that

$$
\overline{\bar{m}}_{0}<m_{0} \text { and } \overline{\bar{m}}_{0} \in\left[\bar{M}, M_{\infty}\right] \text { and } \overline{\bar{h}}_{0}=h_{0}=\mathcal{H}\left(\overline{\bar{m}}_{0}\right) \leq \bar{H} \text {. }
$$

Of course, we take $\overline{\bar{h}}_{0}=h_{0}$. To prove the existence of $\overline{\bar{m}}_{0} \in\left[\bar{M}, M_{\infty}\right]$, it suffices to show that $h_{0} \in\left[\mathcal{H}\left(M_{\infty}\right), \bar{H}\right]$. Indeed, we know from Lemma 10 , and especially (32) that the function $\mathcal{H}:\left[\bar{M}, M_{\infty}\right] \rightarrow\left[\mathcal{H}\left(M_{\infty}\right), \bar{H}\right]$ is $C^{1}$ and strictly decreasing. Now, from (47), we deduce that

- if $M_{\infty}=1$, then $m_{0} \leq M_{\infty}=1$ and $\mathcal{H}\left(m_{0}\right)<h_{0} \leq \bar{H}$; as the function $\mathcal{H}$ is strictly decreasing, we conclude that $\mathcal{H}\left(M_{\infty}\right) \leq \mathcal{H}\left(m_{0}\right)<$ $h_{0} \leq \bar{H}$, hence that $h_{0} \in\left[\mathcal{H}\left(M_{\infty}\right), \bar{H}\right]$;

- if $M_{\infty}<1$, then $\mathcal{H}\left(M_{\infty}\right)=0$ by $(32)$; as $0 \leq h_{0} \leq \bar{H}$, we conclude that $0=\mathcal{H}\left(M_{\infty}\right)<h_{0} \leq \bar{H}$, hence that $h_{0} \in\left[\mathcal{H}\left(M_{\infty}\right), \bar{H}\right]$.

Notice in passing that, as the function $\mathcal{H}$ is strictly decreasing, we have that

$$
\overline{\bar{m}}_{0}=\bar{M} \Longleftrightarrow \overline{\bar{h}}_{0}=h_{0}=\bar{H} .
$$

We have proved that $\overline{\bar{m}}_{0}$ exists and that $\overline{\bar{m}}_{0} \in\left[\bar{M}, M_{\infty}\right]$. There remains to show that $\overline{\bar{m}}_{0}<m_{0}$. Now, from $\overline{\bar{m}}_{0} \in\left[\bar{M}, M_{\infty}\right]$ and $\overline{\bar{h}}_{0}=h_{0}=\mathcal{H}\left(\overline{\bar{m}}_{0}\right)$, we deduce that $\left(\overline{\bar{m}}_{0}, \overline{\bar{h}}_{0}\right)=\left(\overline{\bar{m}}_{0}, h_{0}\right) \in \mathbb{V}$, defined in (34). By definition (34) of $\mathbb{V}$, using the property that the function $\mathcal{H}$ is strictly decreasing, we have that

$$
\left(\overline{\bar{m}}_{0}, \overline{\bar{h}}_{0}\right)=\left(\overline{\bar{m}}_{0}, h_{0}\right) \in \mathbb{V} \Rightarrow\left(m, h_{0}\right) \in \mathbb{V}, \forall m \leq \overline{\bar{m}}_{0} .
$$

As $\left(m_{0}, h_{0}\right) \notin \mathbb{V}$ by assumption (46), we conclude that $\overline{\bar{m}}_{0}<m_{0}$.

From now on, we suppose that $h_{0}<\bar{H}$, so that, summing up the properties shown above, the point $\left(\overline{\bar{m}}_{0}, \overline{\bar{h}}_{0}\right)$ is such that

$$
\left.\left.\overline{\bar{m}}_{0}<m_{0} \text { and } \overline{\bar{m}}_{0} \in\right] \bar{M}, M_{\infty}\right] \text { and } \overline{\bar{h}}_{0}=h_{0}=\mathcal{H}\left(\overline{\bar{m}}_{0}\right)<\bar{H} \text {. }
$$


Now, in addition to the trajectory $t \mapsto(m(t), h(t))$ that is the solution to (4) for the control trajectory $u(\cdot)$ when $(m(0), h(0))=\left(m_{0}, h_{0}\right)$, we study the trajectories

- $t \mapsto(\overline{\bar{m}}(t), \overline{\bar{h}}(t))$, the solution to (4) when $u(t)=\bar{u}$ and when the starting point is $(\overline{\bar{m}}(0), \overline{\bar{h}}(0))=\left(\overline{\bar{m}}_{0}, \overline{\bar{h}}_{0}\right)=\left(\overline{\bar{m}}_{0}, h_{0}\right)$ as in 50), that is, on the right of the frontier of $\mathbb{V}$ (the bottom frontier of the shaded part in Figure 50;

- $t \mapsto(\bar{m}(t), \bar{h}(t))$, the solution to (4) when $u(t)=\bar{u}$ and when the starting point is $(\bar{m}(0), \bar{h}(0))=\left(m_{0}, h_{0}\right)$.

We have the following inequalities between initial conditions:

$$
(\overline{\bar{m}}(0), \overline{\bar{h}}(0))=\left(\overline{\bar{m}}_{0}, \overline{\bar{h}}_{0}\right) \leq\left(m_{0}, h_{0}\right)=(m(0), h(0))=(\bar{m}(0), \bar{h}(0)) .
$$

By Proposition 6, we deduce that

$$
(\overline{\bar{m}}(t), \overline{\bar{h}}(t)) \leq(\bar{m}(t), \bar{h}(t)) \leq(m(t), h(t)), \quad \forall t \geq 0 .
$$

By Lemma 11, we obtain in particular that there exists $T>0$ such that

$$
\bar{H}=\overline{\bar{h}}(T) \leq \bar{h}(T) \leq h(T) .
$$

Here, $T>0$ because (as can be seen in the proof of Lemma 11),

$$
\overline{\bar{m}}(0)=\overline{\bar{m}}_{0}>\bar{M} \text { and } \overline{\bar{h}}(0)=\overline{\bar{h}}_{0}<\bar{H} .
$$

We prove that $\overline{\bar{h}}(T)<\bar{h}(T)$.

Suppose, by contradiction, that $\overline{\bar{h}}(T)=\bar{h}(T)$. Therefore, the function $t \geq 0 \mapsto \bar{h}(t)-\overline{\bar{h}}(t)$ is nonnegative with a minimum at $T>0$, so that

$$
\frac{d \bar{h}(t)}{d t}_{\mid t=T}-\frac{d \overline{\bar{h}}(t)}{d t}_{\mid t=T}=0 .
$$

From (4), we deduce that

$$
A_{h} \bar{m}(T)(1-\bar{h}(T))-\gamma \bar{h}(T)=A_{h} \overline{\bar{m}}(T)(1-\overline{\bar{h}}(T))-\gamma \overline{\bar{h}}(T) .
$$


As $\overline{\bar{h}}(T)=\bar{h}(T)<1$, we obtain that $\bar{m}(T)=\overline{\bar{m}}(T)$. Therefore, we have

$$
(\overline{\bar{m}}(T), \overline{\bar{h}}(T))=(\bar{m}(T), \bar{h}(T))
$$

This is impossible because the two trajectories $t \mapsto(\overline{\bar{m}}(t), \overline{\bar{h}}(t))$, and $t \mapsto$ $(\bar{m}(t), \bar{h}(t))$ are generated by the same vector fields, so that they must be equal. However, we have $\overline{\bar{m}}(0)=\overline{\bar{m}}_{0}<m_{0}=\bar{m}(0)$.

To conclude, we have proven that, for any control trajectory $u(\cdot)$ as in (5), the state trajectory starting from any point of the set $\mathbb{V}^{0}(\bar{H}) \backslash \mathbb{V}$ does not satisfy the constraint (6) for a time $T<+\infty$.

\subsection{Epidemiological interpretation}

The following theorem summarizes the description of the viability kernel depending on whether the upper limit $\bar{H}$ for the proportion of infected humans in (6) is low, high or medium.

Theorem 14. The viability kernel $\mathbb{V}(\bar{H})$ in $(7)$ is as follows.

L) High constraint, that is, a low threshold of infected humans. If

$$
\bar{H}<\frac{A_{h}-\gamma \bar{u} / A_{m}}{A_{h}+\gamma}
$$

the viability kernel consists only of the origin:

$$
\mathbb{V}(\bar{H})=\{(0,0)\}
$$

H) Weak constraint, that is, a high threshold of infected humans. If

$$
\frac{A_{h}}{A_{h}+\gamma}<\bar{H}
$$

the viability kernel $\mathbb{V}(\bar{H})$ is the whole constraint set, that is,

$$
\mathbb{V}(\bar{H})=\mathbb{V}^{0}(\bar{H})=\{(m, h) \mid 0 \leq m \leq 1,0 \leq h \leq \bar{H}\}=[0,1] \times[0, \bar{H}]
$$


M) Medium constraint, that is, a medium threshold of infected humans. If

$$
0<\frac{A_{h}-\gamma \bar{u} / A_{m}}{A_{h}+\gamma}<\bar{H}<\frac{A_{h}}{A_{h}+\gamma},
$$

the viability kernel $\mathbb{V}(\bar{H})$ is a strict subset of the constraint set $\mathbb{V}^{0}(\bar{H})$ whose upper right frontier is a smooth and decreasing curve,

$$
\mathbb{V}(\bar{H})=([0, \bar{M}] \times[0, \bar{H}]) \bigcup\left\{(m, h) \mid \bar{M} \leq m \leq M_{\infty}, h \leq \mathcal{H}(m)\right\},
$$

where $\mathcal{H}: m \mapsto \mathcal{H}(m)$ is the solution to

$$
\begin{aligned}
-g_{m}(m, \mathcal{H}(m), \bar{u}) \mathcal{H}^{\prime}(m)+g_{h}(m, \mathcal{H}(m)) & =0, \\
\mathcal{H}(\bar{M}) & =\bar{H} .
\end{aligned}
$$

The results of Theorem 14 make sense from an epidemiological point of view.

L) One extreme situation is when the maximum proportion $\bar{H}$ of infected humans is low. In that case, the imposed constraint not to overshoot $\bar{H}$ can only be satisfied when there are no infected humans nor infected mosquitoes at the start.

H) The other extreme situation is when the maximum proportion $\bar{H}$ of infected humans is high. In that case, we are allowing a large fraction of the population to get infected. For any trajectory starting in the so-called constraint set (that is, such that the value with which the proportion of people starts is located below the maximum proportion), any mortality rate due to fumigation will make the proportion of infected humans always below $\bar{H}$ for all times.

M) The most interesting case is when the maximum proportion $\bar{H}$ of infected humans is medium (satisfying the condition $(550)$ ). Here, the viability kernel is the strict subset of the constraint set whose upper frontier matches the state orbit associated with the maximum mortality rate due to fumigation (by Lemma 11). 
Focusing more on this last case, the condition (55) of Theorem 14 can be rewritten as follows:

$$
0<\frac{A_{h}}{\gamma+A_{h}}-\frac{\gamma}{A_{m}\left(\gamma+A_{h}\right)} \bar{u}<\bar{H}<\frac{A_{h}}{\gamma+A_{h}} .
$$

This new form (57) taken by the inequalities (55) allows us to visualize the relation between the maximum proportion $\bar{H}$ of infected humans and the maximum $\bar{u}$ mortality fumigation rate. Figure 6 displays the graph of $\bar{H}$ versus $\bar{u}$.

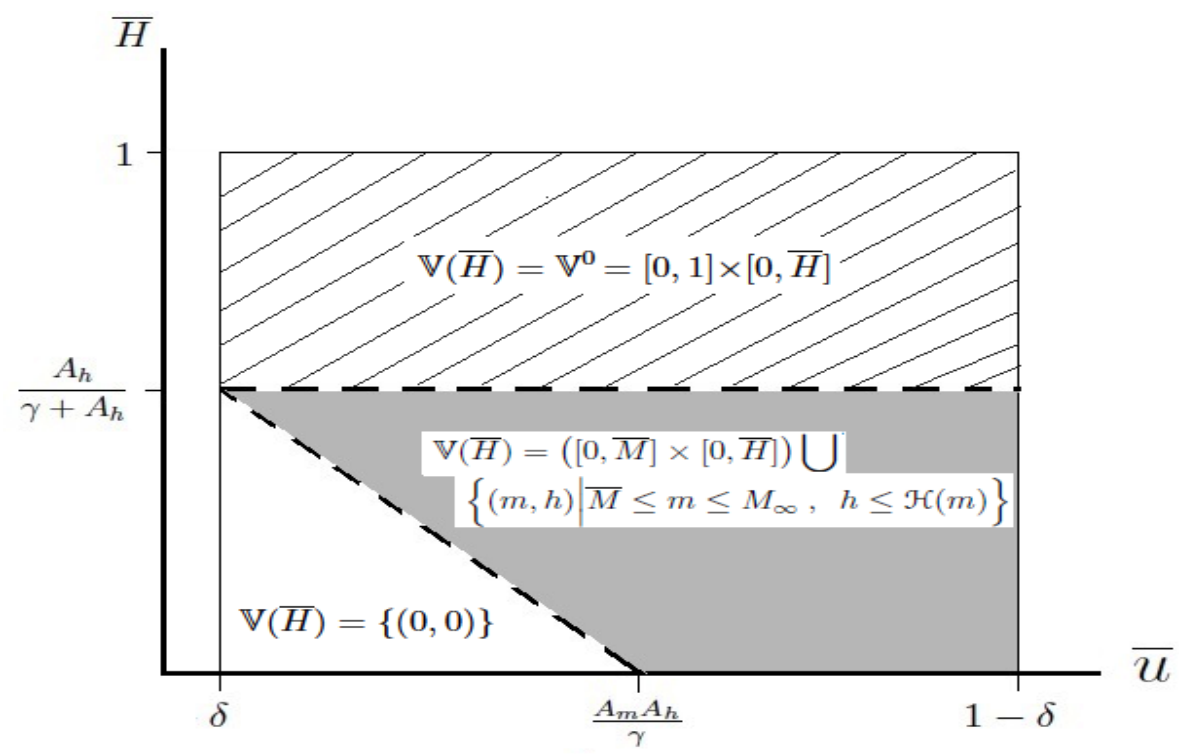

Figure 6: Viability kernel for the Ross-MacDonald model (1), in function of the cap $\bar{H}$ on the proportion of infected humans and of the maximum mortality rate $\bar{u}$ of mosquitoes

Equalities in the inequalities on both sides of (57) correspond to the two dashed straight lines on Figure 6 .

L) When the couple $(\bar{u}, \bar{H})$ belongs to the unshaded bottom triangle (corresponding to a low upper bound $\bar{H}$ ), the viability kernel consists only of the origin.

H) When the couple $(\bar{u}, \bar{H})$ belongs to the upper rectangle shaded with lines (corresponding to a high upper bound $\bar{H}$ ), the viability kernel is the whole constraint set $\mathbb{V}^{0}(\bar{H})=[0,1] \times[0, \bar{H}]$. 
H) When the couple $(\bar{u}, \bar{H})$ belongs to the the gray shaded region (corresponding to a medium upper bound $\bar{H}$ ), the viability kernel is a strict subset of the constraint set $\mathbb{V}^{0}(\bar{H})=[0,1] \times[0, \bar{H}]$, whose top right frontier is the smooth curve given in (56b).

\subsection{Viable controls}

After having characterized the viability kernel, we turn to discussing socalled viable controls. In viability theory [2], it is proven that any control $u \in[\underline{u}, \bar{u}]$, such that the vector $\left(g_{m}, g_{h}\right)$ points towards the inside of the viability kernel $\mathbb{V}(\bar{H})$ is viable in the following sense: a trajectory of viable controls and an initial state in $\mathbb{V}(\bar{H})$ produce a state trajectory in $\mathbb{V}(\bar{H})$.

L) With a strong constraint as in (51), it is impossible to keep the proportion of infected humans below $\bar{H}$ for all times, for whatever admissible control trajectory (5) (except if there are no infected humans or mosquitoes at the start), by Proposition 7 .

H) With a weak constraint as in $(53)$, the constraint set $\mathbb{V}^{0}(\bar{H})=[0,1] \times$ $[0, \bar{H}]$ is strongly invariant, by Proposition 8 . Therefore, any admissible control trajectory (5) makes it possible that the proportion of infected humans remains below $\bar{H}$ for all times. For instance, the minimum control $\underline{u}=\delta$, corresponding to natural death rate (hence to no control), is a viable control.

M) With a medium constraint as in (55), a viable control is the stationary control $u(\cdot)=\bar{u}$, corresponding to maximal fumigation. However, other controls might be viable: it suffices that, on the frontier of the viability kernel $\mathbb{V}(\bar{H})$, a control yields a vector $\left(g_{m}, g_{h}\right)$ that points towards the inside of $\mathbb{V}(\bar{H})$. For instance, we could opt for a control which is low when far from the frontier of the viability kernel $\mathbb{V}(\bar{H})$, but reaches $\bar{u}$ when close to the frontier. Letting $d(m, h)$ be the distance between point $(m, h)$ and the upper frontier of the viability kernel $\mathbb{V}(\bar{H})$, we propose the following viable control for problem (2.2):

$$
u(t)=(1-\exp (-d(m(t), h(t)))) \underline{u}+\exp (-d(m(t), h(t))) \bar{u} .
$$

Figures 7 and 8 display examples of viable control and state (infected humans) trajectories for the Ross-MacDonald model (1) with a proportion of $1 \%$ infected humans not to be exceeded $(\bar{H}=0.01)$. 


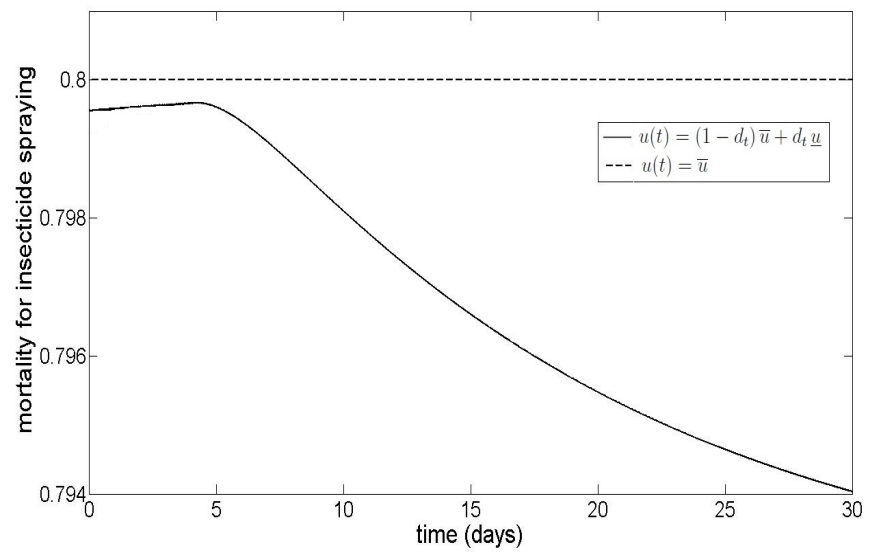

Figure 7: Viable control trajectories for the Ross-MacDonald model (1) with $\bar{H}=0.01$

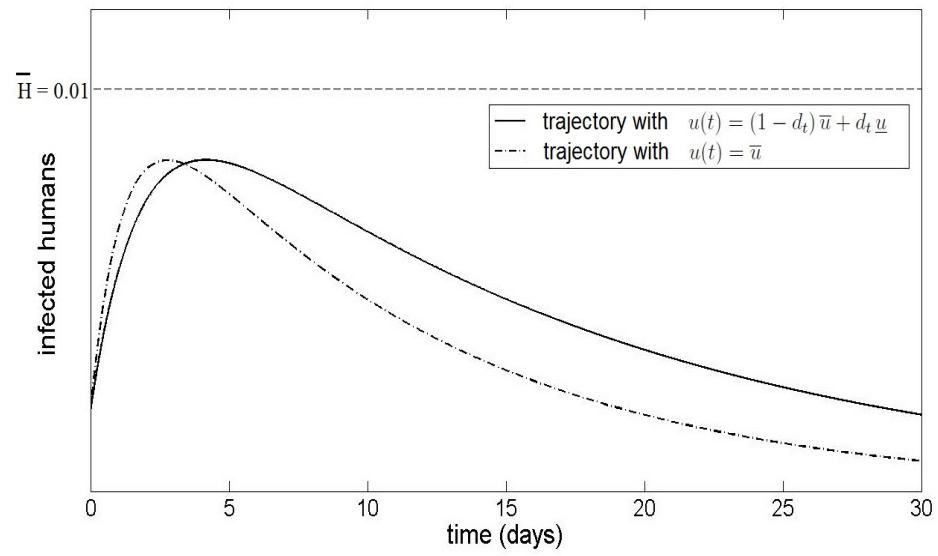

Figure 8: Viable state (infected humans) trajectories for the Ross-MacDonald model (1) with $\bar{H}=0.01$

\section{Conclusion}

As said in the introduction, the approach we have developed is (to our best knowledge) new in mathematical epidemiology. Instead of aiming at an equilibrium or optimizing, we have looked for policies able to maintain 
the infected individuals below a threshold for all times. More precisely, we have allowed for time-dependent fumigation rates to reduce the population of mosquito vector, in order to maintain the proportion of infected individuals by dengue below a threshold for all times. By definition, the viability kernel is the set of initial states (mosquitoes and infected individuals) for which such a fumigation control trajectory exists.

Our theoretical results are the following. For the Ross-Macdonald model with vector mortality control, we have been able to characterize the viability kernel associated with the viability constraint that consists in capping the proportion of infected humans for all times. Depending on whether the cap on the proportion of infected is low, high or medium, we have provided different expressions of the viability kernel. We have also characterized so-called viable policies that produce, at each time, a fumigation rate as a function of current proportions of infected humans and mosquitoes, such that the proportion of infected humans remains below a threshold for all times.

Regarding the use of our theoretical results, we have provided a numerical application in the case of the control of a dengue outbreak in 2013 in Cali, Colombia. Indeed, thanks to numerical data yielded by the Municipal Secretariat of Public Health of Cali, we have produced figures of viability kernels and of viable trajectories. What our analysis suggests is that, to cap the proportion of infected humans at the peak, you need to measure the proportion of infected mosquitoes, at least when it is larger than the characteristic proportion $\bar{M}$, defined in $(28)$. Measuring mosquito abundance is a difficult task, whereas measuring a proportion of infected mosquitoes might be done by proper sampling. Naturally, the weaker the control, the more you need to estimate the proportion of infected mosquitoes, as illustrated in Figure 9. 


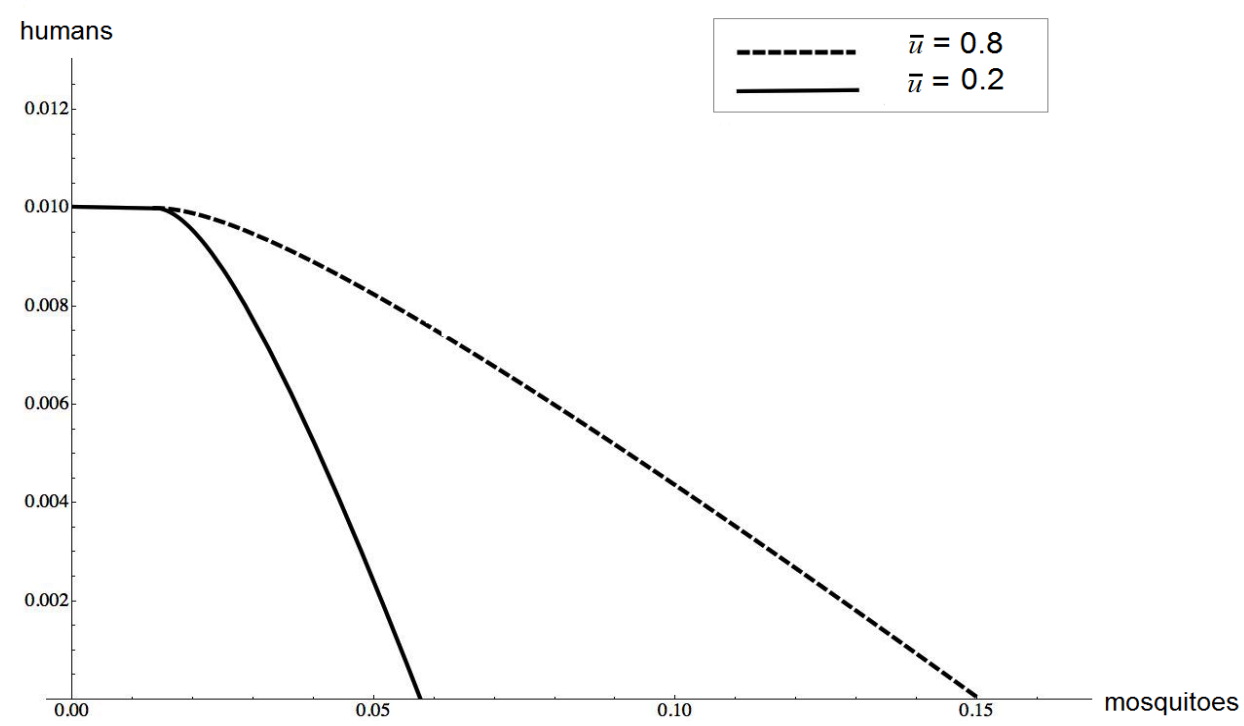

Figure 9: Dependence of the viability kernel on the maximal fumigation mortality rate $\bar{u}$

\section{A Appendix}

\section{A.1 Recalls on comparison theorems for differential systems}

Definition 15. The function $g: \mathbb{R}^{n} \rightarrow \mathbb{R}^{n}$ is said to be quasi monotonous if $g$ is $C^{1}$ and that

$$
\frac{\partial g^{i}}{\partial x_{j}} \geq 0, \quad \forall i \neq j .
$$

In what follows, all inequalities between vectors have to be understood componentwise.

Theorem 16. Let $f$ and $g$ be two vector fields on $D \subset \mathbb{R}^{n}$, with $f$ or $g$ quasi monotonous and $f \leq g$. Suppose that the differential systems

$$
\dot{\mathbf{x}}=f(\mathbf{x}), \dot{\mathbf{y}}=g(\mathbf{y}),
$$

have solutions $t \mapsto \mathbf{x}_{t}$ and $t \mapsto \mathbf{y}_{t}$ defined for all $t \geq 0$. Then, if $\mathbf{x}_{0} \leq \mathbf{y}_{0}$, we have that $\mathbf{x}_{t} \leq \mathbf{y}_{t}$ for all $t \geq 0$. 
Proof. We consider two cases.

(a) Suppose that $f<g$ - that is, $f^{i}<g^{i}$ for all $i=1, \ldots, n$ - and that $\mathbf{x}_{0}<\mathbf{y}_{0}$. We define

$$
\tau=\inf \left\{t \geq 0 \mid \exists i=1, \ldots, n, \mathbf{x}_{t}^{i}>\mathbf{y}_{t}^{i}\right\}
$$

We show that $\tau=+\infty$, that is, $\mathbf{x}_{t} \leq \mathbf{y}_{t}$ for all $t \geq 0$. Indeed, let us suppose the contrary. If $\tau<+\infty$, then there exists at least one $i=1, \ldots, n$ such that

$$
\mathbf{x}_{\tau}^{i}=\mathbf{y}_{\tau}^{i}, \mathbf{x}_{\tau}^{j} \leq \mathbf{y}_{\tau}^{j}, \forall j \neq i
$$

Supposing that $g$ is quasi monotonous (the proof is similar when $f$ is quasi monotonous), we deduce that $g^{i}\left(\mathbf{x}_{\tau}\right) \leq g^{i}\left(\mathbf{y}_{\tau}\right)$.

Moreover, as $\mathbf{x}_{t}^{i}>\mathbf{y}_{t}^{i}$, for all $\left.t \in\right] \tau, \tau+\epsilon\left[\right.$ (for $\epsilon$ small enough) and $\mathbf{x}_{\tau}^{i}=\mathbf{y}_{\tau}^{i}$, we have that

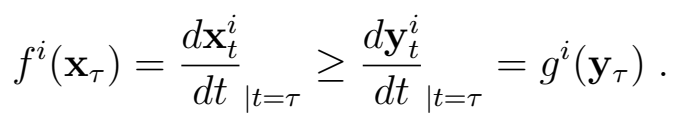

From $g^{i}\left(\mathbf{x}_{\tau}\right) \leq g^{i}\left(\mathbf{y}_{\tau}\right), g^{i}\left(\mathbf{y}_{\tau}\right) \leq f^{i}\left(\mathbf{x}_{\tau}\right)$ and $f^{i}<g^{i}$, we deduce that

$$
g^{i}\left(\mathbf{x}_{\tau}\right) \leq g^{i}\left(\mathbf{y}_{\tau}\right) \leq f^{i}\left(\mathbf{x}_{\tau}\right)<g^{i}\left(\mathbf{x}_{\tau}\right) .
$$

This is contradictory. As a consequence, $\mathbf{x}_{0}<\mathbf{y}_{0}$ implies that $\mathbf{x}_{t} \leq \mathbf{y}_{t}$ for all $t \geq 0$.

(b) Suppose that $f \leq g$ and $\mathbf{x}_{0} \leq \mathbf{y}_{0}$. For any $\epsilon>0$, denote by $\mathbf{y}_{t}^{\epsilon}$ the solution of

$$
\dot{\mathbf{y}^{\epsilon}}=g\left(\mathbf{y}^{\epsilon}\right)+\epsilon, \quad \mathbf{y}_{0}^{\epsilon}=\mathbf{y}_{0}+\epsilon .
$$

We have that

$$
\mathbf{x}_{0}<\mathbf{y}_{0}^{\epsilon}, \quad f<g+\epsilon .
$$

Therefore, we can conclude from the previous item that $\mathbf{x}_{t} \leq \mathbf{y}_{t}^{\epsilon}$ for all $t \geq 0$. It is well known that, for fixed $t$, when $\epsilon \downarrow 0$, we have that $\mathbf{y}_{t}^{\epsilon} \rightarrow \mathbf{y}_{t}$. As a consequence, $\mathbf{x}_{0} \leq \mathbf{y}_{0}$ implies that $\mathbf{x}_{t} \leq \mathbf{y}_{t}$ for all $t \geq 0$.

The following extension is immediate. 
Theorem 17. Let $\left(f_{t}\right)_{t \geq 0}$ and $\left(g_{t}\right)_{t \geq 0}$ be two families of vector fields on $D \subset$ $\mathbb{R}^{n}$, with $f_{t}$ or $g_{t}$ quasi monotonous and $f_{t} \leq g_{t}$, for all $t \geq 0$. Suppose that the differential systems

$$
\dot{\mathbf{x}}=f_{t}(\mathbf{x}), \dot{\mathbf{y}}=g_{t}(\mathbf{y}),
$$

have solutions $t \mapsto \mathbf{x}_{t}$ and $t \mapsto \mathbf{y}_{t}$ defined for all $t \geq 0$. Then, if $\mathbf{x}_{0} \leq \mathbf{y}_{0}$, we have that $\mathbf{x}_{t} \leq \mathbf{y}_{t}$ for all $t \geq 0$.

\section{A.2 Epidemic model adjusted to 2013 dengue outbreak in Cali, Colombia}

The city of Cali, in Colombia, has witnessed several episodes of dengue, as displayed in Figure 10.

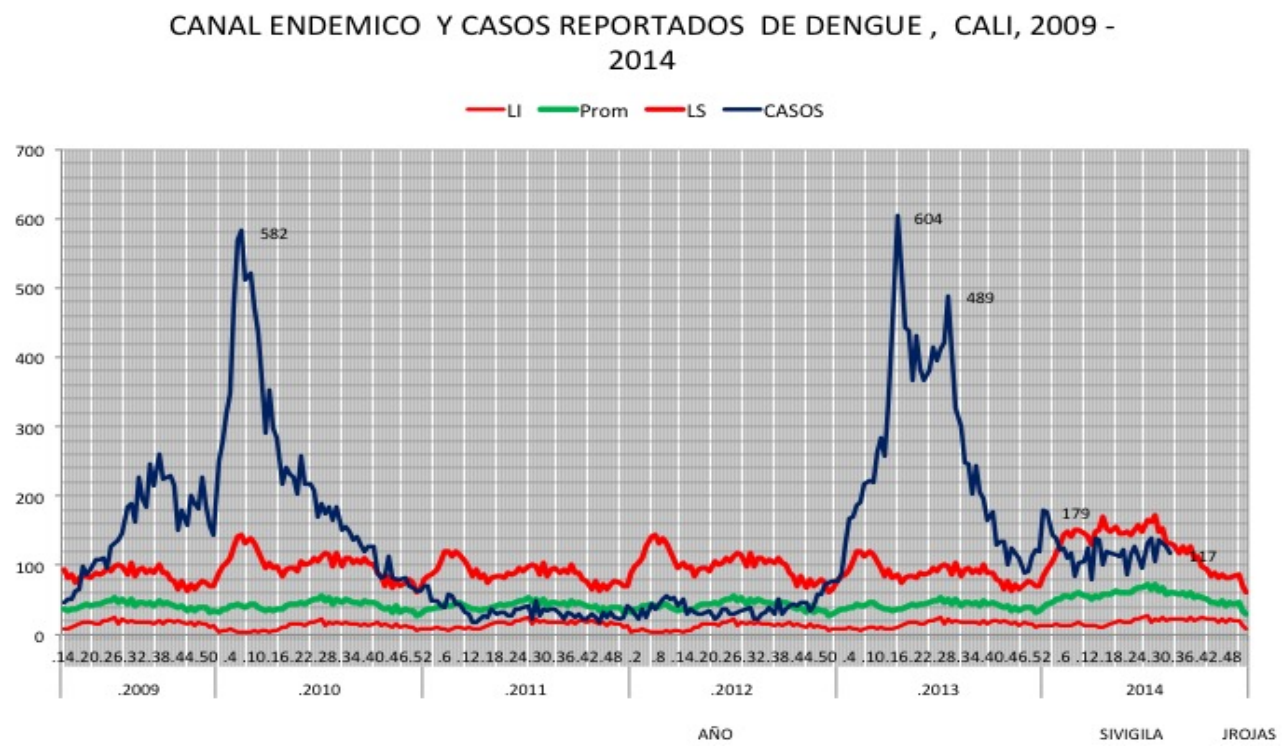

Figure 10: Number of infected by dengue, revealing several episodes of dengue in the city of Cali, in Colombia

The Municipal Secretariat of Public Health of Cali provided us with data corresponding to the dengue outbreak registered in 2013. Here, we present how we have estimated the parameters in the Ross-Macdonal model (1) using the information of daily reports for new registered cases of dengue in Cali - to obtain different figures of viability kernels and of viable trajectories. 


\section{Definition of parameters}

We introduce the state vector

$$
z(t)=(m(t), h(t)) \in[0,1]^{2}
$$

and the vector of parameters

$$
\theta=\left(\alpha, p_{h}, p_{m}, \xi, \delta\right) \in \Theta \subset \mathbb{R}_{+}^{5}
$$

consisting of the five parameters previously defined in Table 1 . The parameter set $\Theta \subset \mathbb{R}_{+}^{5}$ is given by the Cartesian product of the five intervals in the third column of Table 2 .

With these notations, the Ross-Macdonal model (1) now writes

$$
\begin{aligned}
\frac{d}{d t} z(t ; \theta) & =\mathbf{f}(z(t ; \theta), \theta) & & t>0 \\
z(0 ; \theta) & =z_{0} & & z_{0}=\left(m_{0}, h_{0}\right)^{\prime}
\end{aligned}
$$

where

$$
\mathbf{f}(z, \theta)=\left(\begin{array}{c}
f_{1}(z, \theta) \\
f_{2}(z, \theta)
\end{array}\right)=\left(\begin{array}{c}
\alpha p_{m} h(1-m)-\delta m \\
\alpha p_{h} \xi m(1-h)-\gamma h
\end{array}\right), \gamma=0.1
$$

\section{Daily data deduced from health reports}

Notice that the rate $\gamma$ of human recovery does not appear in the parameter vector $\theta$ in $(60)$. Indeed, in the data we only have new cases of dengue registered per day; there is no information regarding how many people recover daily. We chose an infectiousness period of 10 days, that is, a rate of human recovery fixed at $\gamma=0.1$. Under this assumption, the daily incidence data (i.e., numbers of newly registered cases reported on daily basis) provided by the Municipal Secretariat of Public Health (Cali, Colombia) can be converted into the daily prevalence data (i.e., numbers of infected people on a given day, be they new or not). With this, we deduce values of daily proportion of infected people in the form of the set

$$
\mathbb{O}=\left\{\left(t_{j}, \widehat{h}_{j}\right), j=0,1, \ldots, \mathcal{D}\right\},
$$

where $t_{j}$ refers to $j$-th day, within the observation period of $(\mathcal{D}+1)$ days, and where $\widehat{h}_{j}$ stands for the fraction of infected people at the day $t_{j}$. Naturally, 


\begin{tabular}{|c|c|c|c|c|}
\hline Parameter & Initial value & Range & Reference & Estimated value \\
\hline$\alpha$ & 1 & {$[0,5]$} & {$[11,26]$} & 0,3365 \\
\hline$p_{m}$ & 0.5 & {$[0,1]$} & & 0,1532 \\
\hline$p_{h}$ & 0.5 & {$[0,1]$} & & 0,2287 \\
\hline$\xi$ & 1 & {$[1,5]$} & {$[24,27]$} & 1,0359 \\
\hline$\delta$ & 0.035 & {$\left[\frac{1}{30}, \frac{1}{15}\right]$} & {$[11,27]$} & 0.0333 \\
\hline
\end{tabular}

Table 2: Initial values, admissible ranges, respective source references, and estimated values of parameters (numerical solution of optimization problem (64)).

the first couple in the set $(63)$ defines the initial condition $h\left(t_{0}\right)=\widehat{h}_{0}$ with $t_{0}=0$ (initial observation day). Unfortunately, there is no available data for the fraction of infected mosquitoes. As mosquito abundance is strongly correlated with dengue outbreaks [21], we have chosen a linear relation $m(0)=3 \widehat{h}_{0}$ at the beginning of an epidemic outburst (other choices gave similar numerical results).

\section{Parameter estimation procedure}

To estimate a parameter vector $\theta \in \Theta$ that suits with the data, we have applied the curve-fitting approach based on least-square method. More precisely, we look for an optimal solution to the problem of constrained optimization

$$
\min _{\theta \in \Theta} \varphi(\theta)=\frac{1}{2} \sum_{j=1}^{\mathcal{D}}\left(h\left(t_{j}, \theta\right)-\widehat{h}_{j}\right)^{2}, \quad \mathcal{D}=60 \text { days },
$$

subject to the differential constraint (61). Regarding numerics, we have solved this optimization problem with the lsqcurvefit routine (MATLAB Optimization Toolbox), starting with an admissible $\theta_{0} \in \Theta$ (see its exact value in Table 2, second column). The routine generates a sequence $\left\{\theta_{1}, \theta_{2}, \ldots\right\}$ that we stop once it is stationary, up to numerical precision. For a better result, we have combined two particular methods (Trust-RegionReflective Least Squares Algorithm [29] and Levenberg-Marquardt Algorithm [25]) in the implementation of the Isqcurvefit MATLAB routine.

The last column of Table 2 provides estimated values for the parameters $\theta=\left(\alpha, p_{h}, p_{m}, \xi, \delta\right)$, and Figure 11 displays the curve-fitting results. 


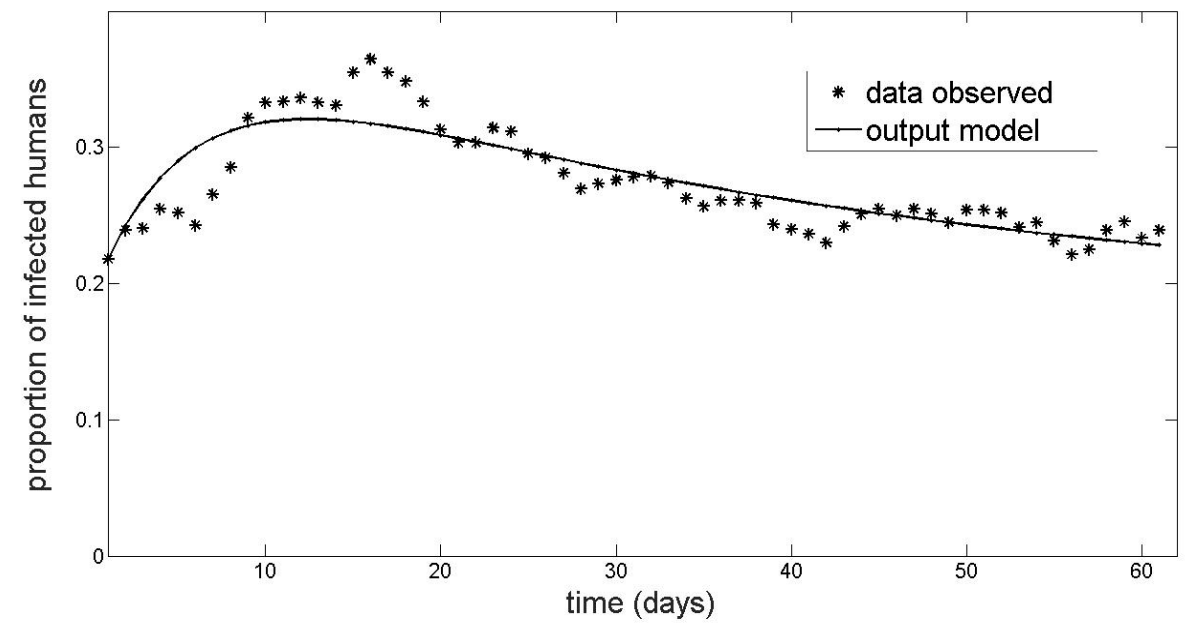

Figure 11: Fraction of people infected with dengue obtained by adjustment of the Ross-Macdonal model (11) (smooth solid curve) versus registered daily prevalence cases (star isolated points) during the 2013 dengue outbreak in Cali, Colombia

Acknowledgments. The authors thank the French program PEERS-AIRD (Modèles d'optimisation et de viabilité en écologie et en économie) and the Colombian Programa Nacional de Ciencias Básicas COLCIENCIAS (Modelos y métodos matemáticos para el co trol y vigilancia del dengue, código 125956933846) that offered financial support for missions, together with École des Ponts ParisTech (France), Université Paris-Est (France), Universidad Autónoma de Occidente (Cali, Colombia) and Universidad del Valle (Cali, Colombia).

\section{References}

[1] R. M. Anderson and R. M. May. Infectious Diseases of Humans: Dynamics and Control. Oxford Science Publications. OUP Oxford, 1992.

[2] J. P. Aubin. Viability theory. Systems \& Control: Foundations \& Applications. Birkhäuser Boston Inc., Boston, MA, 1991. 
[3] C. Béné, L. Doyen, and D. Gabay. A viability analysis for a bio-economic model. Ecological Economics, 36:385-396, 2001.

[4] D. Bertsekas and I. Rhodes. On the minimax reachability of target sets and target tubes. Automatica, 7:233-247, 1971.

[5] G. Bitsoris. On the positive invariance of polyhedral sets for discretetime systems. Systems and Control Letters, 11(3):243-248, 1988.

[6] F. Blanchini. Set invariance in control (survey paper). Automatica, 35(11):1747-1767, 1999.

[7] N. Bonneuil and K. Müllers. Viable populations in a prey-predator system. Journal of Mathematical Biology, 35(3):261-293, February 1997.

[8] N. Bonneuil and P. Saint-Pierre. Population viability in three trophiclevel food chains. Applied Mathematics and Computation, 169(2):1086 - 1105, 2005.

[9] F. Brauer and C. Castillo-Chávez. Mathematical models in population biology and epidemiology, volume 40 of Texts in Applied Mathematics. Springer-Verlag, New York, 2001.

[10] F. H. Clarke, Y. S. Ledayev, R. J. Stern, and P. R. Wolenski. Qualitative properties of trajectories of control systems: a survey. Journal of Dynamical Control Systems, 1:1-48, 1995.

[11] A. Costero, J. D. Edman, G. G. Clark, and T. W. Scott. Life table study of Aedes aegypti (diptera: Culicidae) in Puerto Rico fed only human blood plus sugar. Journal of Medical Entomology, 35(5), 1998.

[12] R. V. Culshaw, S. Ruan, and R. J. Spiteri. Optimal HIV treatment by maximising immune response. Journal of Mathematical Biology, 48(5):545-562, 2004.

[13] M. De Lara and L. Doyen. Sustainable Management of Natural Resources. Mathematical Models and Methods. Springer-Verlag, Berlin, 2008.

[14] O. Diekmann and J. A. P. Heesterbeek. Mathematical Epidemiology of Infectious Diseases. Wiley, Utrecht, Netherland, 2000. 
[15] E. G. Gilbert and K. T. Tan. Linear systems with state and control constraints: the theory and application of maximal output admissible sets. IEEE Transactions on Automatic Control, 36(9):1008-1020, 1991.

[16] P.-O. Gutman and M. Cwikel. Admissible sets and feedback control for discrete-time linear dynamical systems with bounded controls and states. IEEE Transactions on Automatic Control, 31(4):373- 376, 1986.

[17] E. Hansen and T. Day. Optimal control of epidemics with limited resources. Journal of Mathematical Biology, pages 1-29, 2010.

[18] H. W. Hethcote. Optimal ages of vaccination for measles. Mathematical Biosciences, 89(1):29 - 52, 1988.

[19] H. W. Hethcote. The mathematics of infectious diseases. SIAM Review, 42:599-653, 2000.

[20] H. W. Hethcote and P. Waltman. Optimal vaccination schedules in a deterministic epidemic model. Mathematical Biosciences, 18(3-4):365 381, 1973.

[21] C. C. Jansen and N. W. Beebe. The dengue vector Aedes aegypti: what comes next. Microbes and infection, 12(4):272-279, 2010.

[22] D. Kirschner, S. Lenhart, and S. Serbin. Optimal control of the chemotherapy of HIV. Journal of Mathematical Biology, 35(7):775-792, 1997.

[23] I. M. Longini Jr, E. Ackerman, and L. R. Elveback. An optimization model for influenza A epidemics. Mathematical Biosciences, 38(1-2):141 - 157, 1978.

[24] F. Méndez, M. Barreto, J. F. Arias, G. Rengifo, J. Muñoz, M. E. Burbano, and B. Parra. Human and mosquito infections by dengue viruses during and after epidemics in a dengue-endemic region of Colombia. $\mathrm{Am}$ J Trop Med Hyg., 74(4):678-683, 2006.

[25] J. J. Moré. The Levenberg-Marquardt algorithm: implementation and theory. In Numerical analysis, pages 105-116. Springer, 1978. 
[26] T. W. Scott, P. Amerasinghe, A. C. Morrison, L. H. Lorenz, G. G. Clark, D. Strickman, P. Kittayapong, and J. D. Edman. Longitudinal studies of Aedes aegypti (Diptera: Culicidae) in Thailand and Puerto Rico: blood feeding frequency. Journal of medical entomology, 37(1):89-101, 2000.

[27] T. W. Scott, A. C. Morrison, L. H. Lorenz, G. G. Clark, D. Strickman, P. Kittayapong, H. Zhou, and J. D. Edman. Longitudinal studies of Aedes aegypti (Diptera: Culicidae) in Thailand and Puerto Rico: population dynamics. Journal of medical entomology, 37(1):77-88, 2000.

[28] D. L. Smith, F. E. McKenzie, R. W. Snow, and S. I. Hay. Revisiting the basic reproductive number for malaria and its implications for malaria control. PLoS Biol, 5(3):e42, 022007.

[29] D. C. Sorensen. Newton's method with a model trust region modification. SIAM Journal on Numerical Analysis, 19(2):409-426, 1982.

[30] W. Walter. Ordinary Differential Equations. Graduate Texts in Mathematics. Springer New York, 1998. 